\title{
ANALYSE DE RÉCESSION ET RÉSULTATS DE STABILITÉ D'UNE CONVERGENCE VARIATIONNELLE, APPLICATION À LA THÉORIE DE LA DUALITÉ EN PROGRAMMATION MATHÉMATIQUE
}

\begin{abstract}
Driss Mentagui ${ }^{1}$
Résumé. Soit $X$ un espace de Banach de dual topologique $X^{\prime} \cdot \mathcal{C}(X)$ (resp. $\mathcal{C}\left(X^{\prime}\right)$ ) désigne l'ensemble des parties non vides convexes fermées de $X$ (resp. $w^{*}$-fermées de $X^{\prime}$ ) muni de la topologie de la convergence uniforme sur les bornés des fonctions distances. Cette topologie se réduit à celle de la métrique de Hausdorff sur les convexes fermés bornés [16] et admet en général une représentation en terme de cette dernière [11]. De plus, la métrique qui lui est associée s'est révélée très adéquate pour l'étude quantitative de la stabilité et l'approximation des solutions d'une large classe de problèmes en optimisation convexe [6-9]. Dans cet article, nous montrons que, sous des conditions de qualification naturelles, la stabilité de la convergence associée à la topologie définie sur $\mathcal{C}(X)$ (resp. $\mathcal{C}\left(X^{\prime}\right)$ ) est conservée par une classe de transformations linéaires. En identifiant ensuite toute fonction convexe à son épigraphe et en se basant sur la version ensembliste de la stabilité, nous montrons que la convergence précitée est stable par certaines opérations de l'analyse convexe dont le rôle est fondamental en optimisation et en théorie de la dualité. L'hypothèse clé dans les conditions de qualification assurant la stabilité au niveau fonctionnel, est la notion d'inf-locale compacité d'une fonction convexe, introduite dans [28] et qui se traduit dans l'espace $X^{\prime}$ par la quasi-continuité de sa conjuguée. Nous généralisons ainsi les résultats de stabilité de McLinden et Bergstrom [31] puis ceux de Beer et Lucchetti [17] en dimension infinie. Notre étude s'achève enfin par une application à la théorie de la dualité en programmation mathématique dans le cas d'un espace de Banach non nécessairement réflexif.
\end{abstract}

\begin{abstract}
Let $X$ be a Banach space and $X^{\prime}$ its continuous dual. $\mathcal{C}(X)$ (resp. $\mathcal{C}\left(X^{\prime}\right)$ ) denotes the set of nonempty convex closed subsets of $X$ (resp. $w^{*}$-closed subsets of $X^{\prime}$ ) endowed with the topology of uniform convergence of distance functions on bounded sets. This topology reduces to the Hausdorff metric topology on the closed and bounded convex sets [16] and in general has a Hausdorff-like presentation [11]. Moreover, this topology is well suited for estimations and constructive approximations [6-9]. We prove here, that under natural qualification conditions, the stability of the convergence associated to the topology defined on $\mathcal{C}(X)$ (resp. $\mathcal{C}\left(X^{\prime}\right)$ ) is preserved by a class of linear transformations. Building on these results, and by identifing each convex function with its epigraph, the stability at the functional level is acquired towards some operations of convex analysis which play a basic role in convex optimization and duality theory. The key hypothesis in the qualification conditions ensuring the functional stability is the notion of inf-local compactness of a convex function introduced in [28] and expressed in the space $X^{\prime}$ by the quasi-continuity of its conjugate. Then we generalize the stability results of McLinden and Bergstrom [31] and the ones of Beer and Lucchetti [17] in infinite dimension case. Finally we give some applications in convex optimization and mathematical programming in general Banach spaces.
\end{abstract}

Classification Mathématique. 49A50, 49B50, 26A51, 54B20.

Reçu le 19 Septembre 2001. Révisé le 21 décembre 2002.

Mots Clés. Fonction convexe, opérateur linéaire, convergence au sens d'Attouch-Wets, Mosco/épi-convergence, convergence uniforme sur les bornés, inf-(locale) compacité, quasi-continuité, cône (fonction) horizon, dualité, stabilité, approximation et optimisation.

1 Laboratoire d'Analyse Convexe et Variationnelle, Systèmes Dynamiques et Processus Stochastiques, Université Ibn Tofail, Faculté des Sciences, Département de Mathématiques, BP. 133, Kenitra, Maroc; e-mail : d_mentagui@hotmail.com 


\section{INTRODUCTION}

Soient $\mathcal{C}\left(\mathbb{R}^{p}\right)$ l'ensemble des convexes fermés non vides de $\mathbb{R}^{p}$ et $\Gamma_{0}\left(\mathbb{R}^{p}\right)$ l'ensemble des fonctions convexes propres et sci (semi-continues inférieurement) définies sur $\mathbb{R}^{p}$ à valeurs dans $\overline{\mathbb{R}}$. Une des convergences naturelles qu'on peut définir sur $\mathcal{C}\left(\mathbb{R}^{p}\right)$, fut introduite pour la première fois par Wijsman dans le cadre de l'analyse non linéaire $[48,49]$. L'introduction de cette convergence en optimisation a été motivée par certaines questions en théorie de la décision statistique [20,48]. Cette convergence correspond à la convergence simple des fonctions distances : une suite $\left(C_{n}\right)_{n}$ de $\mathcal{C}\left(\mathbb{R}^{p}\right)$ converge vers $C \in \mathcal{C}\left(\mathbb{R}^{p}\right)$ et on note $C_{n} \rightarrow C$ si et seulement si $d(x$, $\left.C_{n}\right) \rightarrow d(x, C), \forall x \in R^{p}$, ce qui est encore équivalent à la convergence des $C_{n}$ vers $C$ au sens de PainlevéKuratowski $[29,42]$. Une des propriétés remarquables de cette convergence est qu'elle conserve la continuité de l'application de polarité $C \in \mathcal{C}\left(\mathbb{R}^{p}\right) \rightarrow C^{0} \in \mathcal{C}\left(\mathbb{R}^{p}\right)$ (voir [49]), où $C^{0}$ désigne l'ensemble polaire de $C$. En d'autres termes, si $C_{n} \rightarrow C$ alors $C_{n}^{0} \rightarrow C^{0}$. Ceci se traduit lorsqu'on identifie une fonction à son épigrahe, par la continuité de l'application $f \in \Gamma_{0}\left(\mathbb{R}^{p}\right) \rightarrow f^{*} \in \Gamma_{0}\left(\mathbb{R}^{p}\right)$, où $f^{*}$ désigne la transformée de LegendreFenchel de $f$. Autrement dit, si $f_{n}, f$ sont des fonctions de $\Gamma_{0}\left(\mathbb{R}^{p}\right)$ et si $f_{n} \rightarrow f$, i.e. epi $f_{n} \rightarrow$ epif alors epi $f_{n}^{*} \rightarrow$ epi $f^{*}$, i.e. $f_{n}^{*} \rightarrow f^{*}$. La convergence des $f_{n}$ en ce sens s'appelle épi-convergence dans la littérature. Cette convergence a pris un bel essor depuis son introduction par Wijsman en 1964, et depuis lors, de nombreux travaux ont été consacrés à l'étude de cette convergence et à ses prolongements en dimension infinie, car elle s'est révélée un outil efficace et puissant dans l'étude de nombreux problèmes, comme ceux relevant de la théorie de l'approximation [1,23], des inéquations variationnelles [36, 37,44], de l'optimisation paramétrique et stochastique ainsi que la programmation mathématique $[1,12,23,46]$. C'est ainsi que Mosco a introduit dans [36-38] une nouvelle convergence qui conserve la continuité de l'application $f \in \Gamma_{0}(X) \stackrel{\Phi}{\longrightarrow} f^{*} \in \Gamma_{0}\left(X^{\prime}\right)$ ( $X$ étant un Banach réflexif de dual topologique $X^{\prime}$ ), et qui se réduit à l'épi-convergence en dimension finie. Soit $\mathcal{C}(X)$ l'ensemble des convexes fermés non vides de $X$. On dira qu'une suite $\left(C_{n}\right)_{n}$ de $\mathcal{C}(X)$ converge au sens de Mosco vers un ensemble $C$ de $\mathcal{C}(X)$ si :

(i) $\forall x \in C \quad \exists x_{n} \in C_{n}: \quad x_{n} \stackrel{\|\cdot\|}{\longrightarrow} x$;

(ii) $\forall\left(n_{k}\right)_{k}$ une sous-suite, si $\overrightarrow{x_{k}} \in C_{n_{k}}$ et $x_{k} \stackrel{w}{\longrightarrow} x$ alors $x \in C$.

Pour plus de détails sur cette convergence et ses applications on pourra consulter $[1,13,28,37,38]$. Malheureusement cette convergence ne conserve pas la continuité de l'application $\Phi$ dans le cas non réflexif, et ne se réduit pas en général à la convergence de Hausdorff sur les bornés de $X$ [16]; de plus il s'est avéré qu'elle est inadéquate pour étudier la stabilité d'une classe de problèmes en optimisation convexes $[16,17,23]$, ce qui a conduit à introduire une nouvelle convergence plus forte que la convergence au sens de Mosco mais qui conserve la continuité de $\Phi$ dans un espace normé quelconque et qui se réduit à l'épi-convergence en dimension finie. Cette convergence possède en outre des propriétés remarquables sans que $X$ soit réflexif. Elle correspond à la convergence uniforme des fonctions distances $d\left(., C_{n}\right)$ vers $d(., C)$ sur les bornés de $X$, et admet une représentation en termes de la distance de Hausdorff $[11,16,17]$. Cette convergence s'est révélée aussi très adéquate pour l'étude quantitative de la stabilité des solutions d'une large classe de problèmes d'optimisation régularisés ou perturbés [6-9], ainsi que pour l'étude de la stabilité des problèmes d'optimisation bien posés [16,17,23].

L'objet de cet article est de montrer que, sous des conditions de qualifications naturelles, cette dernière convergence est stable par certaines opérations classiques de l'analyse convexe qui jouent un rôle fondamental tant en optimisation convexe qu'en théorie de l'approximation. L'hypothèse clé dans ces conditions de qualifications, est la notion d'inf-locale compacité d'une fonction convexe qui se traduit dans l'espace dual par la quasi-continuité de sa fonction conjuguée [28]. Ainsi, nous prolongeons les résultats de stabilité de Beer et Lucchetti [17], puis ceux de McLinden et Bergstrom [31]. Notre étude s'achève par une application à la théorie de la dualité en programmation mathématique dans un cadre non nécessairement réflexif.

\section{Préliminatres}

Soit $X$ un espace de Banach muni de la topologie de sa norme |.| (appelée aussi topologie forte) ou de sa topologie faible $\sigma\left(X, X^{\prime}\right), X^{\prime}$ étant son dual topologique. On désigne par $\mathcal{B}(0, r)$ la boule fermée de centre 0 et de rayon $r>0$. 
Pour une fonction $f \in \overline{\mathbb{R}}^{X}$, on note respectivement le domaine effectif de $f$ et son épigraphe par :

$$
\text { Dom } f=\{x \in X / f(x)<+\infty\} \text {, epi } f=\{(x, \alpha) \in X \times \mathbb{R} / f(x) \leq \alpha\} .
$$

On rappelle que $f$ est semi-continue inférieurement sur $X$ (ou brièvement sci) si et seulement si epi $f$ est fermé dans $X \times \mathbb{R} . f$ est dite propre si elle ne prend jamais la valeur $-\infty$ et prend au moins une valeur finie. La conjuguée de $f$ ou sa transformée de Legendre-Fenchel dans la dualité $\left(X, X^{\prime}\right)$ est la fonction notée $f^{*}$ définie pour tout $x^{\prime} \in X^{\prime}$ par $f^{*}\left(x^{\prime}\right)=\sup \left\{\left\langle x, x^{\prime}\right\rangle-f(x) / x \in \operatorname{Dom} f\right\}[24,30]$. Le sous-différentiel de $f$ au sens de l'analyse convexe en un point $x \in X$, noté $\partial f(x)$, est l'ensemble éventuellement vide, défini par, $\partial f(x)=\left\{x^{\prime} \in X^{\prime} / f^{*}\left(x^{\prime}\right)+f(x)=\left\langle x, x^{\prime}\right\rangle\right\}$. Le sous-différentiel de $f$ noté $\partial f$, est le graphe de la multifonction $x \in X \longrightarrow \partial f(x) \subset X^{\prime}$, c'est-à-dire, $\partial f=\left\{\left(x, x^{\prime}\right) \in X \times X^{\prime} / x^{\prime} \in \partial f(x)\right\}$.

Si $C$ est un ensemble non vide de $X$, on désigne par :

- $(f, C)$ le problème de minimisation : trouver $\bar{x} \in C$ tel que $f(\bar{x})=\inf (f, C)$;

- $\arg \min (f, C)=\{\bar{x} \in C / f(\bar{x})=\inf (f, C)\}$;

- $\varepsilon \cdot \arg \min (f, C)=\{x \in X / f(x) \leq \inf (f, C)+\varepsilon\}$ si $\varepsilon>0$ et $\inf (f, C) \in \mathbb{R}$.

Le problème $(f, C)$ est dit bien posé au sens de Tikhonov (resp. au sens de Levitin-Polyak) [23] si arg min $(f, C)$ est réduit à un singleton $x_{0}$ et si toute suite $\left(x_{n}\right)_{n}$ de $C$ telle que $f\left(x_{n}\right) \rightarrow \inf (f, C)$ (resp. si toute suite $\left(x_{n}\right)_{n}$ de $X$ telle que $d\left(x_{n}, C\right) \rightarrow 0$ et $\left.f\left(x_{n}\right) \rightarrow \inf (f, C)\right)$ converge vers $x_{0}$. Pour l'intérêt de ces notions et leur rôle crucial en optimisation et en théorie d'approximation on peut consulter $[16,17,23,32]$. La fonction indicatrice de $C$, notée $\delta_{C}$, est la fonction qui vaut 0 sur $C$ et $+\infty$ ailleurs. On note int $C$, ir $C$ et $\bar{C}$ respectivement l'intérieur de $C$, l'intérieur de $C$ relativement à la variété affine engendrée par $C$ et la fermeture de $C$. Si de plus $C$ est convexe et fermé, on note par $C^{\infty}$ le cône horizon de $C$ [42] (appelé aussi cône de récesssion ou cône asymptote de $C[30,40]$ selon la terminologie classique). Son expression est donnée par $C^{\infty}=\cap_{\varepsilon>0} \varepsilon\left(C-x_{0}\right)$, avec $x_{0}$ un élément de $C$. On vérifie classiquement que $C^{\infty}$ ne dépend pas du choix de $x_{0}$ dans $C$, et si $f$ est une fonction convexe propre sci, il existe une fonction et une seule notée $f^{\infty}$ telle que (epi $\left.f\right)^{\infty}=$ epi $f^{\infty}$. Cette fonction est appelée la fonction horizon de $f$ et son expression est donnée par :

$$
f^{\infty}(x)=\sup _{\lambda>0} \frac{f\left(x_{0}+\lambda x\right)-f\left(x_{0}\right)}{\lambda} \text {, où } x_{0} \in \operatorname{Dom} f .
$$

Notons que cette fonction ne dépend pas du choix de $x_{0}$ dans $\operatorname{Dom} f$ et $f^{*} \infty=\left(\delta_{\operatorname{Dom} f}\right)^{*}$ [30]. L'épi-somme de deux fonctions $f$ et $g$ est la fonction notée $f$ \# $g$ et définie pour chaque $x \operatorname{par}(f \# g)(x)=\inf \{f(u)+g(x-u)$ : $u \in X\}$. Pour l'intérêt de cette opération on peut consulter $[2,5,30,35]$.

Soit $\tau$ une topologie définie sur $X$ de sorte que $(X, \tau)$ soit un espace vectoriel topologique localement convexe séparé (e.l.c.s.), et soit $V$ un voisinage de 0 convexe fermé équilibré associé à cette topologie. On note par $j_{V}$ la jauge de $V$, i.e. la fonction définie sur $X$ par $j_{V}(x)=\inf \{\alpha>0: x \in \alpha V\}$, avec la convention inf $\emptyset=+\infty$. On vérifie aisément que $j_{V}$ est une semi-norme $\tau$-continue et $V=\left\{x / j_{V}(x) \leq 1\right\}[19,21,27]$.

Soient maintenant $f_{n}, f$ des fonctions convexes propres sci définies sur $X$ et $C_{n}, C$ des convexes fermés non vides de $X$. On dit que,

1. $C_{n}$ converge au sens de Mosco vers $C$ [36-38] et on note $C_{n} \stackrel{M}{\longrightarrow} C$ si

a) $\forall x \in C \exists x_{n} \in C_{n}: x_{n} \rightarrow x$ ( $\rightarrow$ désigne la convergence au sens de la norme);

b) $\forall\left(n_{k}\right)_{k}$ une sous-suite, si $x_{k} \in C_{n_{k}}$ et $x_{k} \rightarrow x$ alors $x \in C$ ( $\rightarrow$ désigne la convergence faible).

On dit que $f_{n}$ converge au sens de Mosco vers $f[1,38]$ et on note $f_{n} \stackrel{M}{\longrightarrow} f$ si epi $f_{n} \stackrel{M}{\longrightarrow}$ epi $f$ dans $X \times \mathbb{R}$, ce qui est encore équivalent à (voir [1]) :

$\left.\mathrm{a}^{\prime}\right) \forall x \in X, \forall x_{n} \rightarrow x, f(x) \leq \underline{\lim } f_{n}\left(x_{n}\right)$;

$\left.\mathrm{b}^{\prime}\right) \forall x \in X, \exists x_{n} \rightarrow x, f_{n}\left(x_{n}\right) \rightarrow f(x)$.

On dit que $f_{n}$ est slice convergente vers $f$ et on note $f_{n} \stackrel{\tau_{s}}{\longrightarrow} f[4]$ si :

$\left.\mathrm{a}^{\prime \prime}\right) \forall x \in X, \exists x_{n} \rightarrow x, f_{n}\left(x_{n}\right) \rightarrow f(x)$; 
$\left.\mathrm{b}^{\prime \prime}\right) \forall y \in X^{\prime}, \exists y_{n} \stackrel{\|\cdot\|_{X^{\prime}}}{\longrightarrow} y, f_{n}^{*}\left(y_{n}\right) \rightarrow f^{*}(y)$,

ce qui est encore équivalent d'après la même référence aux deux conditions suivantes :

i) $\partial f_{n} \rightarrow \partial f$, càd : $\forall(x, y) \in \partial f, \exists\left(x_{n}, y_{n}\right) \in \partial f_{n}$ tel que $\left(x_{n}, y_{n}\right) \rightarrow(x, y)$; et pour toute sous-suite $\left(n_{k}\right)_{k}$ si $\left(x_{k}, y_{k}\right) \in \partial f_{n_{k}}$ et $\left(x_{k}, y_{k}\right) \rightarrow(x, y)$ alors $(x, y) \in \partial f$;

ii) $\exists(x, y) \in \partial f, \exists\left(x_{n}, y_{n}\right) \in \partial f_{n}$ tels que $\left(x_{n}, f_{n}\left(x_{n}\right), y_{n}\right) \rightarrow(x, f(x), y)$;

2. $C_{n}$ converge vers $C$ au sens d'Attouch-Wets [6-9] et on note $C_{n} \stackrel{A W}{\longrightarrow} C \sin \sup _{|x| \leq \rho}\left|d\left(x, C_{n}\right)-d(x, C)\right| \rightarrow$ $0 \forall \rho>0$, ce qui est encore équivalent [11] à $\operatorname{Haus}_{\rho}\left(C_{n}, C\right) \rightarrow 0$ où

$$
\operatorname{Haus}_{\rho}\left(C_{n}, C\right)=\max \left\{e\left(C_{n} \cap \mathcal{B}(0, \rho), C\right), e\left(C \cap \mathcal{B}(0, \rho), C_{n}\right)\right\}
$$

avec $e(A, B)=\sup \{d(x, B), x \in A)\}$. On note $f_{n} \stackrel{A W}{\longrightarrow} f$ si epi $f_{n} \stackrel{A W}{\longrightarrow}$ epi $f$ dans $X \times \mathbb{R}$.

Dans un espace normé quelconque, la convergence d'Attouch-Wets est plus fine que la slice convergence et que cette dernière est plus fine que la Mosco convergence [45]. En dimension finie ces trois convergences coïncident [4, $6,15]$ et note alors $f_{n} \stackrel{e}{\longrightarrow} f$ et $C_{n} \rightarrow C$. Pour plus de détails sur ces convergences et leur rôle crucial en optimisation et en théorie d'approximation on peut consulter $[1,6,10,16,17,23,25]$. Une étude plus détaillée en dimension finie de la stabilité d'une classe importante de convergences d'ensembles et de fonctions, ainsi que leur caractérisation, se trouvent dans la référence [42].

\section{VERSION ENSEMBLISTE DE LA STABILITÉ}

L'objet de cette section et de montrer que la convergence d'ensembles au sens d'Attouch-Wets est stable par la transformation d'opérateurs linéaires lorsque certaines conditions de qualification sont satisfaites. On étudiera en particulier la stabilité de la somme d'ensembles convexes par cette convergence.

Théorème 3.1. Soient $X$ et $Y$ deux espaces de Banach, $A_{n}, A: X \longrightarrow Y$ des opérateurs linéaires continus et $C_{n}, C$ des convexes fermés non vides de $X$, avec $C$ fortement ou faiblement localement compact. Supposons que

a) $\left\|A_{n}-A\right\| \longrightarrow 0, C_{n} \stackrel{A W}{\longrightarrow} C$; Alors

b) $\operatorname{Ker} A \cap C^{\infty}=\{0\}$.

1. $A_{n} C_{n} \stackrel{A W}{\longrightarrow} A C$;

2. $\operatorname{Ker} A_{n} \cap C_{n}^{\infty}=\{0\}$, pour $n$ suffisamment grand;

3. si pour $n$ suffisamment grand, $C_{n}$ est fortement ou faiblement localement compact, alors $A_{n} C_{n}$ est fermé. En particulier ceci aura lieu si $X$ est réflexif et si $C$ est faiblement localement compact.

Pour la preuve de ce théorème, nous utiliserons les lemmes suivants :

Lemme 3.1. [16] Soient $X$ un espace normé et $C_{n}, C$ des convexes fermés de $X$, avec $C$ borné et $C_{n} \stackrel{A W}{\longrightarrow} C$. Alors les $C_{n}$ sont uniformément bornés :

$$
\exists N \in \mathbb{N}, \exists r>0, \forall n \geq N, C_{n} \subset \mathcal{B}(0, r) .
$$

Lemme 3.2. [17] Soit $X$ un espace normé et soient $C_{n}, C$ et $D_{n}, D$ des convexes fermés de $X$ tels que $C_{n} \stackrel{A W}{\longrightarrow} C, D_{n} \stackrel{A W}{\longrightarrow} D$ et $C$ nint $D \neq \emptyset$. Alors $C_{n} \cap D_{n} \stackrel{A W}{\longrightarrow} C \cap D$.

Lemme 3.3. [21, 22] Soient $X$ un espace localement convexe séparé, $C$ un convexe fermé localement compact contenant 0 et $V$ un voisinage de 0 , convexe fermé équilibré tel que $V \cap C$ soit un compact. On désigne par $j$ la jauge de $V$. Alors, pour toute suite généralisée $\left(x_{i}\right)_{i \in I}$ de $C$, on a

- ou bien $\left(j\left(x_{i}\right)\right)_{i \in I}$ est bornée et $\left(x_{i}\right)_{i \in I}$ admet une sous-famille filtrée convergente dans $C$;

- ou bien $\left(j\left(x_{i}\right)\right)_{i \in I}$ n'est pas bornée et $\left(\frac{x_{i}}{j\left(x_{i}\right)}\right)_{i \in I}$ admet une sous-famille filtrée convergente vers un élément non nul de $C^{\infty}$. 
Lemme 3.4. Soient $X, Y$ deux espaces localement convexes séparés, $A: X \longrightarrow Y$ un opérateur linéaire continu et $C$ un convexe fermé de $X$, localement compact. Supposons que $\operatorname{Ker} A \cap C^{\infty}$ soit un sous-espace vectoriel. Alors $A C$ est fermé.

Démonstration. Par translation, on peut supposer sans nuire à la généralité, que $0 \in C$.

Étape 1. Supposons que $M=\operatorname{Ker} A \cap C^{\infty}=\{0\}$. Soit $y \in \overline{A C}$, il existe une suite généralisée $\left(x_{i}\right)_{i \in I}$ de $C$ telle que $A x_{i} \longrightarrow y$. Soit $V$ un voisinage de 0 convexe fermé équilibré tel que $V \cap C$ soit un compact et soit $j$ la jauge de $V$. Si $\left(j\left(x_{i}\right)\right)_{i \in I}$ n'est pas bornée, on peut supposer que $j\left(x_{i}\right) \longrightarrow+\infty$. Il existe d'après le lemme 3.3 , une sous-famille filtrée, notée encore $\left(x_{i}\right)_{i \in I}$ telle que $\frac{x_{i}}{j\left(x_{i}\right)} \longrightarrow x \in C^{\infty}$ et $x \neq 0$. Par suite $\frac{A x_{i}}{j\left(x_{i}\right)} \longrightarrow A x=0$. Ainsi, $x \in \operatorname{Ker} A \cap C^{\infty}=\{0\}$, ce qui est absurde. Donc $\left(j\left(x_{i}\right)\right)_{i \in I}$ est bornée, et par le lemme $3.3,\left(x_{i}\right)_{i \in I}$ admet une sous-famille filtrée convergente vers un élément $x$ de $C$. Comme $A$ est continu, $y=A x$. Donc $A C$ est fermé.

Étape 2. Supposons que $M$ soit non nul. On va se ramener à l'étape précédente par passage à l'espace quotient $X / M$. En effet, considérons les applications canoniques $\phi: X \longrightarrow X / M, \phi(x)=\dot{x}$ et $\dot{A}: X / M \longrightarrow Y$ avec $\dot{A}(\dot{x})=A x$. L'application $\dot{A}$ est bien définie car $M \subset \operatorname{Ker} A$; de plus elle est linéaire et continue. Posons $\dot{C}=\phi(C)$ et $\dot{V}=\phi(V)$. Il est clair que $\dot{C}$ est convexe et $\dot{V}$ est un voisinage de 0 . D'autre part, nous avons :

i) $\dot{C}$ est un convexe fermé : pour cela, il suffit de montrer que $C$ est saturé, i.e. $C=\phi^{-1}(\phi(C))$. En effet, soit $z \in \phi^{-1}(\phi(C))$, il existe $x \in C$ tel que $z-x \in M$. Comme $M \subset C^{\infty}$, alors $z=x+(z-x) \in C$;

ii) $\dot{C}$ est localement compact : soit $\dot{z} \in \dot{C} \cap \dot{V}$. Il existe $x \in C$ et $u \in V$ tels que $z-x \in M$ et $u-z \in M$. Comme $M \subset C^{\infty}$, nous avons $z \in C$ et $u \in C$. Ainsi, $u \in C \cap V$ et $\dot{z} \in \phi(C \cap V)$. On en déduit que $\dot{C} \cap \dot{V}=\phi(C \cap V)$ est un compact;

iii) $\operatorname{Ker} \dot{A} \cap \dot{C}^{\infty}=\{0\}$ : en effet, soit $\dot{x}$ tel que $\dot{A} \dot{x}=0$ et $\dot{x} \in \dot{C}^{\infty}=\cap_{\epsilon \succ 0} \epsilon \dot{C}$. Alors $A x=0$, et pour tout $\varepsilon>0$, il existe $y \in C$ tel que $\frac{x}{\varepsilon}-y \in M$. Par suite, $\frac{x}{\varepsilon} \in C$. Il en résulte que $x \in \operatorname{Ker} A \cap C^{\infty}=M$. D'où $\dot{x}=0$.

Toutes les hypothèses de l'étape 1 sont donc satisfaites, par suite $A C=\dot{A} \dot{C}$ est un fermé.

Lemme 3.5. Sous les hypothèses du théorème 3.1, il existe des nombres $\alpha>0, \beta>0$ et $N \in \mathbb{N}$ tels que :

$$
\left|A_{n} x\right| \geq \alpha j(x)-\beta, \quad \forall n \geq N, \quad \forall x \in C_{n}
$$

où $j$ désigne la jauge d'un voisinage $V$ de 0 , convexe fermé équilibré tel que $\left(V+x_{0}\right) \cap C$ soit fortement ou faiblement compact, et $x_{0}$ un élément quelconque de $C$.

Démonstration. Puisque $C_{n} \stackrel{A W}{\longrightarrow} C$, on peut supposer sans nuire à la généralité, que $0 \in C_{n} \cap C$. On prendra alors $x_{0}=0$. Raisonnons par l'absurde : supposons qu'il existe une sous-suite $\left(n_{k}\right)_{k}, x_{k} \in C_{n_{k}}$ telle que

$$
\left|A_{n_{k}}\left(x_{k}\right)\right|<\frac{j\left(x_{k}\right)}{k}-1
$$

- Si $\left(j\left(x_{k}\right)\right)_{k}$ est bornée, alors limsup $\left|A_{n_{k}}\left(x_{k}\right)\right| \leq-1$, ce qui est absurde.

- Si $\left(j\left(x_{k}\right)\right)_{k}$ n'est pas bornée, on peut supposer que $j\left(x_{k}\right) \longrightarrow+\infty$ pour une sous-suite notée encore $j\left(x_{k}\right)$. En posons $u_{k}=\frac{x_{k}}{j\left(x_{k}\right)}$, l'équation (3.1) devient

$$
\left|A_{n_{k}}\left(u_{k}\right)\right|<\frac{1}{k}-\frac{1}{j\left(x_{k}\right)} .
$$

D'autre part, la convexité des $C_{n}$ et le fait que $j\left(u_{k}\right)=1$ impliquent que $u_{k} \in C_{n_{k}} \cap V$ pour $k$ suffisamment grand. Comme $C_{n} \stackrel{A W}{\longrightarrow} C$ et $C \cap$ int $V$ est non vide, alors $C_{n} \cap V \stackrel{A W}{\longrightarrow} C \cap V$ par le lemme 3.2. $C \cap V$ étant en plus borné, les ensembles $C_{n} \cap V$ sont donc uniformément bornés par le lemme 3.1. Ainsi, la suite $\left(u_{k}\right)_{k}$ est bornée. Il existe alors une suite $z_{k} \in C \cap V$ telle que $\left|u_{k}-z_{k}\right| \longrightarrow 0$, et par compacité $\left(u_{k}\right)_{k}$ admet une 
sous-suite convergente vers un élément $z$ de $C$. Comme $j$ est continue, on a $j(z)=1$. Donc $z$ est non nul. D'autre part, l'hypothèse $\left\|A_{n}-A\right\| \longrightarrow 0$ et l'inégalité (3.2) impliquent $A z=0$. Soit maintenant $\varepsilon>0$. Pour $k$ suffisamment grand, $\frac{u_{k}}{\varepsilon} \in C_{n_{k}}$. Comme $C_{n} \stackrel{A W}{\longrightarrow} C$ et $\left(\frac{u_{k}}{\varepsilon}\right)_{k}$ converge vers $\frac{z}{\varepsilon}$ (fortement ou faiblement) alors $z \in \varepsilon C$. Il en résulte que $z \in \operatorname{Ker} A \cap C^{\infty}=\{0\}$, ce qui est absurde.

Démonstration du théorème 3.1. On peut supposer sans nuire à la généralité que $0 \in C_{n} \cap C$.

1. Il s'agit de montrer :

a) $\sup _{y \in A_{n} C_{n} \cap \mathcal{B}(0, \rho)} d(y, A C) \longrightarrow 0$ et b) $\sup _{y \in A C \cap \mathcal{B}(0, \rho)} d\left(y, A_{n} C_{n}\right) \rightarrow 0 \forall \rho>0$;

a) d'après le lemme 3.1 , il existe $N \in \mathbb{N}$ et $r>0$ tels que $C_{n} \cap V \subset \mathcal{B}(0, r)$ pour tout $n \geq N$. D'autre part, le lemme 3.5 implique l'existence de deux nombres $\alpha, \beta$ strictement positifs et $N_{1} \in \mathbb{N}$ tels que

$$
\left|A_{n} x\right| \geq \alpha j(x)-\beta, \quad \forall n \geq N_{1}, \quad \forall x \in C_{n} .
$$

Soient $\rho>0, \varepsilon>0, a>\max \left(\frac{\beta+\rho}{\alpha}, 1\right)$ et $\rho^{\prime} \geq a r$. Comme $\left\|A_{n}-A\right\| \rightarrow 0$ et $\sup _{x \in C_{n} \cap \mathcal{B}\left(0, \rho^{\prime}\right)} d(x, C)$ $\rightarrow 0$, il existe $N_{2} \in \mathbb{N}$ tel que pour tout $n \geq N_{2}$, on a

$$
\sup _{|x| \leq \rho^{\prime}}\left|A_{n} x-A x\right| \leq \frac{\varepsilon}{\|A\|+1}
$$

et

$$
\forall x \in C_{n} \cap \mathcal{B}\left(0, \rho^{\prime}\right), \exists u \in C:|x-u| \leq \frac{\varepsilon}{\|A\|+1} .
$$

Choisissons $N^{\prime} \in \mathbb{N}$ tel que $N^{\prime}>\max \left(N, N_{1}, N_{2}\right)$. Soient $n \geq N^{\prime}$ et $y \in A_{n} C_{n} \cap \mathcal{B}(0, \rho)$, avec $y=A_{n} x$ et $x \in C_{n}$. Par (3.3), nous avons :

$$
\rho \geq|y| \geq \alpha j(x)-\beta
$$

d'où

Par suite,

$$
j(x) \leq \frac{\beta+\rho}{\alpha}<a .
$$

et

$$
\frac{x}{a} \in C_{n} \cap V \subset \mathcal{B}(0, r)
$$

$$
x \in C_{n} \cap \mathcal{B}\left(0, \rho^{\prime}\right) .
$$

Mais, d'après (3.5), il existe $u \in C$ tel que $|x-u| \leq \frac{\varepsilon}{\|A\|+1}$. En utilisant (3.4) et (3.6) on obtient

$$
\begin{aligned}
d(y, A C) & \leq|y-A u| \leq\left|A_{n} x-A x\right|+|A x-A u| \\
& \leq \frac{\varepsilon}{\|A\|+1}+\|A\||x-u| \\
& \leq \frac{\varepsilon}{\|A\|+1}+\|A\| \cdot \frac{\varepsilon}{\|A\|+1}=\varepsilon ;
\end{aligned}
$$

b) soient $r^{\prime}$, s et $d$ des nombres strictement positifs tels que $C \cap V \subset \mathcal{B}\left(0, r^{\prime}\right), s \geq a r^{\prime}$ et $d>\sup _{n}\left\|A_{n}\right\|$. Comme $\sup _{x \in C \cap \mathcal{B}(0, s)} d\left(x, C_{n}\right) \rightarrow 0$ et $\left\|A_{n}-A\right\| \rightarrow 0$, il existe $N^{\prime \prime}$ tel que pour tout $n \geq N^{\prime \prime}$ nous avons

et

$$
\sup _{|x| \leq \rho}\left|A_{n} x-A x\right| \leq \frac{\varepsilon}{d+1}
$$

$$
\forall x \in C \cap \mathcal{B}(0, s), \exists u \in C_{n}:|x-u| \leq \frac{\varepsilon}{d+1} .
$$

D'autre part, la convergence des $C_{n}$ vers $C$ et l'inégalité (3.3) impliquent

$$
|A x| \geq \alpha j(x)-\beta, \quad \forall x \in C .
$$


Soient $n \geq N^{\prime \prime}$ et $y \in A C \cap \mathcal{B}(0, \rho)$ avec $y=A x$ et $x \in C$. D'après (3.9) nous avons

$$
\rho \geq|y| \geq \alpha j(x)-\beta .
$$

Donc

$$
x \in C \cap \mathcal{B}(0, s) .
$$

Mais d'après (3.8), il existe $u \in C_{n}$ tel que $|x-u| \leq \frac{\varepsilon}{d+1}$. En utilisant (3.7) et (3.10) on obtient

$$
\begin{aligned}
d\left(y, A_{n} C_{n}\right) & \leq\left|y-A_{n} u\right| \leq\left|A x-A_{n} x\right|+\left|A_{n} x-A_{n} u\right| \\
& \leq \frac{\varepsilon}{d+1}+\left\|A_{n}\right\| \cdot \frac{\varepsilon}{d+1} \\
& \leq \frac{\varepsilon}{d+1}+d \cdot \frac{\varepsilon}{d+1}=\varepsilon ;
\end{aligned}
$$

2. posons $M_{n}=\operatorname{Ker} A_{n} \cap C_{n}^{\infty}$ et $P_{n}=\left\{x \in M_{n} / j(x)>0\right\}$. Comme $j \geq 0, M_{n}=\left\{x \in M_{n} / j(x)=0\right\} \cup$ $P_{n}$. Si $x \in M_{n}$ et $j(x)=0$, alors $x \in C_{n}^{\infty}$ et $x \in \varepsilon V$ pour tout $\varepsilon>0$. Par suite, $x \in C_{n}^{\infty} \cap V^{\infty}=$ $\left(C_{n} \cap V\right)^{\infty}$. Or, pour $n$ assez grand, $C_{n} \cap V$ est borné, donc $\left(C_{n} \cap V\right)^{\infty}=\{0\}$ (voir [40,41]). Ainsi, $M_{n}=\{0\} \cup P_{n}$. Il suffit maintenant de montrer que $P_{n}$ est vide pour $n$ assez grand. Si non, il existe une sous-suite $\left(n_{k}\right)_{k}$ et une suite $\left(x_{k}\right)_{k}$ telles que $x_{k} \in \operatorname{Ker} A_{n_{k}} \cap C_{n_{k}}^{\infty}$ et $j\left(x_{k}\right)>0$. La suite $z_{k}=\frac{x_{k}}{j\left(x_{k}\right)}$ vérifie $A_{n_{k}}\left(z_{k}\right)=0$ et $z_{k} \in C_{n_{k}} \cap V$. Comme les ensembles $C_{n} \cap V$ sont uniformément bornés, la suite $\left(z_{k}\right)_{k}$ est bornée, et selon un argument déjà utilisé, elle admet une sous-suite convergente vers un élément non nul $z \in C$. Nous montrons aussi selon ce même argument (moyennant l'hypothèse $\lambda z_{k} \in C_{n_{k}}, \forall \lambda>0$, $\forall k$ ) que $z \in \operatorname{Ker} A \cap C^{\infty}$, ce qui est absurde. Donc, $\operatorname{Ker} A_{n} \cap C_{n}^{\infty}=\{0\}$ pour $n$ assez grand;

3. soit $N \in \mathbb{N}$ tel que $\operatorname{Ker} A_{n} \cap C_{n}^{\infty}=\{0\}$ pour tout $n \geq N$. Si $C_{n}$ est fortement ou faiblement localement compact pour $n \geq N$, le lemme 3.4 implique que $A_{n} C_{n}$ est fermé. En particulier, si $X$ est réflexif et $C$ est faiblement localement compact, le fait que $C_{n} \cap V$ soit fermé et borné pour $n$ suffisamment grand, implique que cet ensemble est faiblement compact, i.e. $C_{n}$ est faiblement localement compact [19].

Remarque 3.1. La conclusion du théorème 3.1 n'est plus vraie lorsqu'on remplace la convergence au sens d'Attouch-Wets par la convergence au sens de Mosco :

Soient $X=L^{2}([0,2 \pi]), C_{n}=\left\{\alpha e_{n} / \alpha \in \mathbb{R}\right\}, e_{n}(x)=\sin n x, n \geq 1$ et $A_{n}=A: f \in X \longmapsto \int_{0}^{2 \pi} x f(x) \mathrm{d} x$. On vérifie aisément que $C_{n} \stackrel{M}{\longrightarrow} C=\{0\}, A_{n} C_{n}=\mathbb{R}$ et $A C=\{0\}$.

Lorsque $A_{n}=A:(x, y) \in X \times X \longrightarrow x+y \in X$, on obtient un résultat de stabilité analogue à celui du théorème 3.1, mais avec des hypothèses plus faibles. Plus précisément, nous avons

Théorème 3.2. Soit $X$ un Banach et soient $C_{n}, C$ et $D_{n}, D$ des convexes fermés de $X$ tels que $C$ soit fortement ou faiblement localement compact, $C_{n} \stackrel{A W}{\longrightarrow} C$ et $D_{n} \stackrel{A W}{\longrightarrow} D$. Supposons que $C^{\infty} \cap-D^{\infty}=\{0\}$. Alors

1) $C_{n}+D_{n} \stackrel{A W}{\longrightarrow} C+D$;

2) $C_{n}^{\infty} \cap-D_{n}^{\infty}=\{0\}, \forall n \geq N$;

3) $C_{n}+D_{n}$ est fermé si $n \geq N$ et si $C_{n}$ ou $D_{n}$ est fortement ou faiblement localement compact. En particulier, ceci aura lieu si $X$ est réflexif et si $C$ est faiblement localement compact.

La preuve du théorème 3.2 est quasi-identique à celle du théorème 3.1 moyennant les deux lemmes suivants :

Lemme 3.6. [21] Soient $X$ un espace vectoriel topologique séparé et $C, D$ deux convexes fermés non vides de $X$ tels que $C$ soit localement compact et $C^{\infty} \cap-D^{\infty}=\{0\}$. Alors $C+D$ est fermé.

Lemme 3.7. Sous les hypothèses du théorème 3.2, il existe des nombres $\alpha>0, \beta>0$ et $N \in \mathbb{N}$ tels que pour tout $n \geq N$ on $a$

$$
|x+y| \geq \alpha j(x)-\beta, \quad \forall x \in C_{n}, \quad \forall y \in D_{n}
$$

où $j$ désigne la jauge d'un voisinage $V$ de 0 , convexe fermé équilibré tel que $\left(V+x_{0}\right) \cap C$ soit un compact, et $x_{0}$ un élément de $C$. 
Démonstration. On peut supposer sans nuire à la généralité que $0 \in C_{n} \cap C$ et $0 \in D_{n} \cap D$. Supposons qu'il existe une sous-suite $\left(n_{k}\right)_{k}$ et des suites $x_{k} \in C_{n_{k}}, y_{k} \in D_{n_{k}}$ telles que

$$
\left|x_{k}+y_{k}\right|<\frac{j\left(x_{k}\right)}{k}-1
$$

Si $\left(j\left(x_{k}\right)\right)_{k}$ est bornée, alors limsup $\left|x_{k}+y_{k}\right| \leq-1$, ce qui est absurde.

Si $\left(j\left(x_{k}\right)\right)_{k}$ n'est pas bornée, on peut supposer $j\left(x_{k}\right) \longrightarrow+\infty$. L'inégalité (3.12) s'écrit

$$
\left|\frac{x_{k}}{j\left(x_{k}\right)}+\frac{y_{k}}{j\left(x_{k}\right)}\right|<\frac{1}{k}-\frac{1}{j\left(x_{k}\right)},
$$

avec $u_{k}=\frac{x_{k}}{j\left(x_{k}\right)} \in C_{n_{k}} \cap V$ et $v_{k}=\frac{y_{k}}{j\left(x_{k}\right)} \in D_{n_{k}}$ pour $k$ suffisamment grand. Mais, d'après un raisonnement déjà utilisé, la suite $\left(u_{k}\right)_{k}$ converge (pour une sous-suite) vers un élément non nul $u \in C^{\infty}$. En utilisant la convexité de $D_{n}$, l'inégalité (3.13) et la convergence des $D_{n}$ vers $D$, on déduit que $-u \in D^{\infty}$. Ainsi, $u \in C^{\infty} \cap-D^{\infty}=\{0\}$, ce qui est absurde.

Démonstration du théorème 3.2. On peut supposer sans nuire à la généralité que $0 \in C_{n} \cap C$ et $0 \in D_{n} \cap D$.

1) Il s'agit de montrer :

a) $\sup _{x \in\left(C_{n}+D_{n}\right) \cap \mathcal{B}(0, \rho)} d(x, C+D) \longrightarrow 0$ et b) $\sup _{x \in(C+D) \cap \mathcal{B}(0, \rho)} d\left(x, C_{n}+D_{n}\right) \rightarrow 0 \forall \rho>0$;

a) soient $N_{1} \in \mathbb{N}$ et $r>0$, choisis suffisamment grands de sorte que l'inégalité (3.11) et l'inclusion $C_{n} \cap V \subset \mathcal{B}(0, r)$ soient satisfaites pour tout $n \geq N_{1}$. Soient $\varepsilon>0, \rho^{\prime} \geq$ ar $\left(a>\max \left(\frac{\beta+\rho}{\alpha}, 1\right)\right)$ et $N \geq N_{1}$ tel que pour tout $n \geq N$

$$
\sup _{x \in C_{n} \cap \mathcal{B}\left(0, \rho^{\prime}\right)} d(x, C)<\frac{\varepsilon}{2}, \quad \sup _{y \in D_{n} \cap \mathcal{B}\left(0, \rho+\rho^{\prime}\right)} d(y, D)<\frac{\varepsilon}{2} .
$$

Pour tout $n \geq N$ et tout $z \in\left(C_{n}+D_{n}\right) \cap \mathcal{B}(0, \rho), z=x+y, x \in C_{n}$ et $y \in D_{n}$; on a $\rho \geq \alpha j(x)-\beta$. Donc $x \in C_{n} \cap \mathcal{B}\left(0, \rho^{\prime}\right)$, et par suite $y \in D_{n} \cap \mathcal{B}\left(0, \rho+\rho^{\prime}\right)$. Or, d'après (3.14), il existe $u \in C$ et $v \in D$ tels que $|x-u| \leq \frac{\varepsilon}{2}$ et $|y-v| \leq \frac{\varepsilon}{2}$. D'où

$$
d(z, C+D) \leq|z-u-v| \leq|x-u|+|y-v| \leq \varepsilon
$$

b) se démontre de la même façon, moyennant l'inégalité

$$
|x+y| \geq \alpha j(x)-\beta, \forall x \in C, \forall y \in D
$$

qui est une conséquence immédiate du lemme 3.7 ;

2) l'égalité $C_{n}^{\infty} \cap-D_{n}^{\infty}=\{0\}$ se démontre exactement comme celle du point 2. du théorème 3.1 avec $M_{n}=C_{n}^{\infty} \cap-D_{n}^{\infty}$

3) la conclusion de ce point est une conséquence immédiate du point précédent et du lemme 3.6.

Remarque 3.2. La conclusion du théorème 3.2 n'est plus vraie lorsqu'on remplace la convergence au sens d'Attouch-Wets par la convergence au sens de Mosco. En effet (voir [26]) : soient $X$ un espace de Hilbert de base hilbertienne $\left(x_{n}\right)_{n>1}$ et $C_{n}=\left[x_{1}, x_{n}\right], D_{n}=\left[x_{1},-x_{n}\right]$ des segments. Il est clair que ces deux suites d'ensembles convergent au sens de Mosco vers le compact $\left\{x_{1}\right\}$ ! mais $C_{n}+D_{n}$ ne converge pas au sens de Mosco vers $\left\{2 x_{1}\right\}$ car $\left[0, x_{1}\right] \subset C_{n}+D_{n}$. Le lecteur est vivement invité à consulter le papier très récent [26] où la stabilité de la Slice et de la Mosco convergence a été obtenue sous des conditions de qualifications uniformes.

Corollaire 3.1. Supposons que $X$ soit de dimension finie, $A_{n}, A: X \longrightarrow Y$ sont linéaires continus et $\left|A_{n}(x)-A(x)\right| \longrightarrow 0, \forall x \in X$.

Si $C_{n}, C$ sont des convexes fermés tels que $C_{n} \longrightarrow C$ et $\operatorname{Ker} A \cap C^{\infty}=\{0\}$, alors $A_{n} C_{n} \stackrel{A W}{\longrightarrow} A C$ et $A_{n} C_{n}$, $A C$ sont fermés pour $n$ suffisamment grand. 
Démonstration. En dimension finie, la convergence des $C_{n}$ au sens d'Attouch-Wets est équivalente à leur convergence au sens de Painlevé-Kuratowski [1,6,42]. D'autre part, il est classique de vérifier dans ce cas aussi que la convergence ponctuelle des opérateurs $A_{n}$ est équivalente à leur convergence uniforme sur les bornés. On conclut alors avec le théorème 3.1 .

Lorsque $Y$ est aussi de dimension finie, on retrouve le résultat de stabilité de McLinden et Bergstrom [31] (Th. 3).

Corollaire 3.2 ([31], Cor. 3A). Si $\operatorname{dim} X<+\infty$ et $C_{n}, C$ et $D_{n}, D$ sont des convexes fermés tels que $C_{n} \longrightarrow C$, $D_{n} \longrightarrow D$ et $C^{\infty} \cap-D^{\infty}=\{0\}$, alors $C_{n}+D_{n} \longrightarrow C+D$ et ces ensembles sont fermés pour $n$ suffisamment grand.

Démonstration. Celle-ci est immédiate à partir du théorème 3.2 ou du théorème 3.1.

De légères modifications au niveau des preuves du théorème 3.1 et du théorème 3.2 , et le fait que la boule unité de $X^{\prime}$ est toujours $\sigma\left(X^{\prime}, X\right)$ compacte, permettent d'obtenir une version duale de la stabilité dans le sens suivant :

Théorème 3.3. Soient $A_{n}, A: X^{\prime} \longrightarrow Y^{\prime}$ des opérateurs linéaires continus fortement et faiblement $\left(\sigma\left(X^{\prime}, X\right)\right.$, $\left.\sigma\left(Y^{\prime}, Y\right)\right)$ tels que $\left\|A_{n}-A\right\| \longrightarrow 0$, et soient $C_{n}, C$ des convexes $\sigma\left(X^{\prime}, X\right)$ fermés de $X^{\prime}$ tels que $C$ soit $\sigma\left(X^{\prime}, X\right)$ localement compact, $C_{n} \stackrel{A W}{\longrightarrow} C$ et $\operatorname{Ker} A \cap C^{\infty}=\{0\}$. Alors $A_{n} C_{n} \stackrel{A W}{\longrightarrow} A C$ et $A_{n} C_{n}, A C$ sont $\sigma\left(Y^{\prime}, Y\right)$ fermés.

Théorème 3.4. Soient $C_{n}, C$ et $D_{n}, D$ des convexes $\sigma\left(X^{\prime}, X\right)$ fermés tels que $C_{n} \stackrel{A W}{\longrightarrow} C$ et $D_{n} \stackrel{A W}{\longrightarrow} D$. Supposons que $C$ soit $\sigma\left(X^{\prime}, X\right)$ localement compact et $C^{\infty} \cap-D^{\infty}=\{0\}$. Alors $C_{n}+\overrightarrow{D_{n}} \stackrel{A W}{\longrightarrow} C+\vec{D}$ et ces ensembles sont $\sigma\left(X^{\prime}, X\right)$ fermés.

\section{VERSION FONCTIONNELLE DE LA STABILITÉ}

Commençons d'abord par rappeler certains résultats et définitions qui nous seront utiles dans la suite. On rappellera en particulier la notion d'inf-locale compacité d'une fonction ainsi que sa version duale, qui vont jouer un rôle clé dans cette section.

Definition 4.1. Soient $X$ un espace localement convexe séparé et $f: X \rightarrow \overline{\mathbb{R}}$ une fonction convexe. On dit que :

1) $f$ est continue si elle est continue en chaque point de l'intérieur (supposé non vide) de son domaine effectif;

2) $f$ est quasi-continue si Dom $f$ engendre une variété affine $L_{f}$ de codimension finie et si la restriction de $f$ à $L_{f}$ est continue.

Un ensemble convexe est dit quasi-continu si sa fonction indicatrice est quasi-continue.

Pour l'intérêt que présentent ces notions en optimisation convexe, on peut consulter $[28,30]$.

Definition 4.2 ( [28]). Soient $X$ un e.l.c.s. et $f, g$ deux fonctions convexes. On dit que $f$ et $g$ forment un couple uni si leurs domaines effectifs ne sont que trivialement séparés, c'est-à-dire si un hyperplan fermé sépare les deux domaines, il les contient tous les deux. On dira que deux convexes de $X$ sont unis si leurs fonctions indicatrices forment un couple uni.

En dimension finie, la quasi-continuité d'un convexe est toujours vérifiée, et deux convexes $A$ et $B$ sont unis si et seulement si $\operatorname{ir}(A) \cap \operatorname{ir}(B) \neq \emptyset[40]$.

Definition 4.3. Une fonction $f: X \longrightarrow \overline{\mathbb{R}}$ définie sur un e.l.c.s. est dite inf-localement compacte si et seulement si pour tout $\rho \in \mathbb{R},\{x / f(x) \leq \rho\}$ est localement compact. 
Proposition 4.1 [28]. Soit $f: X \longrightarrow \overline{\mathbb{R}}$ une fonction convexe propre et sci, définie sur un e.l.c.s. Les propriétés suivantes sont équivalentes :

1) epi $f$ est localement compact dans $X \times \mathbb{R}$;

2) $f$ est inf-localement compacte;

3) $\exists \rho>\inf _{x} f(x)$ tel que $\{x / f(x) \leq \rho\}$ soit localement compact.

Théorème 4.1. Soient $X$ un espace de Banach et $f_{n}, f, g_{n}, g$ des fonctions convexes propres sci définies sur X. Supposons que $f_{n} \stackrel{A W}{\longrightarrow} f, g_{n} \stackrel{A W}{\longrightarrow} g$ et $f$ est fortement ou faiblement inf-localement compacte.

Si $\left\{x / f^{\infty}(x)+g^{\infty}(-x) \leq 0\right\}=\{0\}$, alors

1) $f_{n} \# g_{n} \stackrel{A W}{\longrightarrow} f \# g$

2) $\exists N \in \mathbb{N}$ tel que $\left\{x / f_{n}^{\infty}(x)+g_{n}^{\infty}(-x) \leq 0\right\}=\{0\}, \forall n \geq N$;

3) si $X$ est réflexif et $f$ est faiblement inf-localement compacte, alors $f_{n} \# g_{n}$ est sci pour $n$ suffisamment grand et la borne inférieure dans ces fonctions est atteinte en tout point où elle est finie.

Démonstration.

1) D'après la proposition 4.1, $f$ est inf-localement compacte ssi epi $f$ est localement compact dans $X \times \mathbb{R}$. D'autre part, il est classique de vérifier que la condition $\left\{x / f^{\infty}(x)+g^{\infty}(-x) \leq 0\right\}=\{0\}$ est équivalente à (epi $f)^{\infty} \cap-(\text { epi } g)^{\infty}=\{0\}$. Toutes les hypothèses du théorème 3.2 sont donc satisfaites. Par conséquent,

$$
\text { epi } f_{n}+\text { epi } g_{n} \stackrel{A W}{\longrightarrow} \text { epi } f+\text { epi } g \text {. }
$$

Ceci est encore équivalent à

Comme

$$
\overline{\text { epi } f_{n}+\text { epi } g_{n}} \underset{A W}{\longrightarrow} \overline{\text { epi } f+\text { epi } g} \text {. }
$$

alors

$$
\text { epi } f_{n}+\text { epi } g_{n} \subset \text { epi } f_{n} \# g_{n} \subset \overline{\text { epi } f_{n}+\text { epi } g_{n}} \text {; }
$$

$$
\overline{\text { epi } f_{n} \# g_{n}} \stackrel{A W}{\longrightarrow} \overline{\operatorname{epi} f \# g} \text {. }
$$

Ainsi, epi $\overline{f_{n} \# g_{n}} \stackrel{A W}{\longrightarrow}$ epi $f$ \# , i.e. $\overline{f_{n} \# g_{n}} \stackrel{A W}{\longrightarrow} f \# g$ où $\overline{f_{n} \# g_{n}}$ désigne la régularisée sci de $f_{n} \# g_{n}$; ce qui est encore équivalent à $f_{n} \# g_{n} \stackrel{A W}{\longrightarrow} f \# g$.

Le reste est une conséquence immédiate des conclusions 2) et 3) du théorème 3.2 et de l'inclusion (4.1), ainsi que la proposition 6.4 de [28].

Lorsque $\mathrm{X}$ est de dimension finie, on retrouve en particulier le théorème 4 de la référence [31]. Pour d'autres résultats de stabilité en dimension finie vis-à-vis de l'opération épi-somme on pourra consulter [42].

Théorème 4.2. Soient $X$ un Banach et $f_{n}, f, g_{n}, g$ des fonctions convexes propres sci définies sur $X$ telles que $f_{n} \stackrel{A W}{\longrightarrow} f$ et $g_{n} \stackrel{A W}{\longrightarrow} g$. Supposons que

1) $f$ est quasi-continue; Alors

2) $\cup_{\lambda \geq 0} \lambda(\operatorname{Dom} f-\operatorname{Dom} g)=X$.

(a) $f_{n}+g_{n} \stackrel{A W}{\longrightarrow} f+g$, et ces fonctions sont propres pour $n$ suffisamment grand;

(b) $\partial\left(f_{n}+g_{n}\right) \rightarrow \partial(f+g)$;

(c) pour $n$ suffisamment grand, $\partial\left(f_{n}+g_{n}\right)(x)=\partial f_{n}(x)+\partial g_{n}(x) \forall x \in \operatorname{Dom} f_{n} \cap \operatorname{Dom} g_{n}$; et la même égalité a lieu pour les fonctions $f$ et $g$ pour tout $x \in \operatorname{Dom} f \cap \operatorname{Dom} g$.

Pour la preuve, nous utiliserons les lemmes suivants :

Lemme 4.1 ( [28]). Soient $X$ un Banach et $f: X \longrightarrow]-\infty,+\infty]$ une fonction convexe propre sci. Alors $f$ est quasi-continue ssi $f^{*}$ est $\sigma\left(X^{\prime}, X\right)$ inf-localement compacte.

Lemme 4.2 ( [28]). Soient $A$ et $B$ deux convexes unis et $A$ quasi-continu. Alors $i r(A) \cap B$ est non vide. 
Lemme 4.3. Soient $X$ un Banach et $f, g$ deux fonctions convexes propres sci définies sur $X$, avec $f$ quasicontinue.

Les propriétés suivantes sont équivalentes :

1) $f$ et $g$ forment un couple uni;

2) $E=\left(\text { epi } f^{*}\right)^{\infty} \cap-\left(\text { epi } g^{*}\right)^{\infty}$ est un sous-espace vectoriel de $X^{\prime} \times \mathbb{R}$;

3) $F=\left\{x^{\prime} \in X^{\prime} / f^{* \infty}\left(x^{\prime}\right)+g^{* \infty}\left(-x^{\prime}\right) \leq 0\right\}$ est un s.e.v. de $X^{\prime}$;

4) $\bar{M}$ est un s.e.v. de $X$ avec $M=\cup_{\lambda \geq 0} \lambda(\operatorname{Dom} f-\operatorname{Dom} g)$;

5) $M$ est un s.e.v. fermé de $X$.

Si une de ces conditions est satisfaite, alors $\operatorname{proj}_{X^{\prime}} E=F=M^{\perp}$.

Démonstration. D'après [28], les propriétés 1), 2) et 3) sont équivalentes sans aucune hypothèse de quasicontinuité.

L'équivalence entre 3) et 4) provient des égalités $\left(\delta_{\bar{M}}\right)^{*}=\delta_{F}$ et $\left(\delta_{F}\right)^{*}=\delta_{\bar{M}}$.

$4) \Longrightarrow 5)$ : comme $f$ est quasi-continue et Dom $f$, Dom $g$ sont unis, le lemme 4.2 implique que $\operatorname{ir}(\operatorname{Dom} f) \cap$ Dom $g$ est non vide. Quitte à faire une translation, on peut supposer sans nuire à la généralité que $0 \in$ $\operatorname{ir}(\operatorname{Dom} f) \cap \operatorname{Dom} g$.

Notons $L_{f}$ l'espace vectoriel engendré par Dom $f$ et $C_{g}=\cup_{\lambda \geq 0} \lambda$ (Dom $g$ ). Il est facile de vérifier, en utilisant la convexité, que $M=L_{f}-C_{g}$. Soit $s: X \longrightarrow X / L_{f}$ la surjection canonique. Comme $s$ est linéaire et continue et que $X / L_{f}$ est de dimension finie, alors $\overline{s(M)}=s(\bar{M})$ et $\overline{s(M)}$ est un sous-espace vectoriel. Il en est de même pour $s(M)$ car $\operatorname{dim} X / L_{f}<+\infty$. Ainsi, $M=s^{-1}(s(M))$ est un s.e.v. de $X$.

Le reste suit par une vérification classique.

Lemme 4.4 ( [14]). Soient $X$ un espace normé et $f_{n}, f: X \longrightarrow \overline{\mathbb{R}}$ des fonctions convexes propres sci. Alors $f_{n} \stackrel{A W}{\longrightarrow} f$ ssi $f_{n}^{*} \stackrel{A W}{\longrightarrow} f^{*}$.

Démonstration du Théorème 4.2. (a) D'après le lemme 4.1, la condition 1) est équivalente à l'inf-locale compacité $\sigma\left(X^{\prime}, X\right)$ de $f^{*}$, et par le lemme 4.3, la condition 2) équivaut à (epi $\left.f^{*}\right)^{\infty} \cap-\left(\text { epi } g^{*}\right)^{\infty}=\{0\}$. Toutes les hypothèses du théorème 3.4 sont donc satisfaites ; par suite epi $f_{n}^{*}+$ epi $g_{n}^{*} \stackrel{A W}{\longrightarrow}$ epi $f^{*}+$ epi $g^{*}$ et ces sommes sont convexes et $\sigma\left(X^{\prime}, X\right)$ fermées. Il en résulte que $f_{n}^{*} \# g_{n}^{*} \stackrel{A W}{\longrightarrow} f^{*} \# g^{*}$ et ces fonctions sont convexes et $\sigma\left(X^{\prime}, X\right)$ sci. Or, pour $n$ assez grand (epi $\left.f_{n}^{*}\right)^{\infty} \cap-\left(\text { epi } g_{n}^{*}\right)^{\infty}=\{0\}$ et $f_{n}^{*}$ est $\sigma\left(X^{\prime}, X\right)$ inf-localement compacte. Ainsi, $f_{n}$ et $g_{n}$ sont unies d'après le lemme 4.3 et $f_{n}$ est quasi-continue d'après le lemme 4.1. Donc Dom $f_{n} \cap$ Dom $g_{n}$ est non vide par le lemme 4.2 . Il en résulte que $f_{n}^{*} \# g_{n}^{*}$ est propre. Il en est de même pour $f^{*} \# g^{*}$. Ainsi $\left(f_{n}+g_{n}\right)^{*}=\left(f_{n}^{*} \# g_{n}^{*}\right)^{* *}=f_{n}^{*} \# g_{n}^{*}$ et $(f+g)^{*}=\left(f^{*} \# g^{*}\right)^{* *}=f^{*} \# g^{*}$. Par suite $\left(f_{n}+g_{n}\right)^{*} \stackrel{A W}{\longrightarrow}(f+g)^{*}$, et par le lemme $4.4, f_{n}+g_{n} \stackrel{A W}{\longrightarrow} f+g$.

(b) D'après (a) $f_{n}+g_{n} \stackrel{A W}{\longrightarrow} f+g$ et ces fonctions sont convexes propres et sci. Comme la slice convergence est moins fine que la convergence d'Attouch-Wets [18,45] alors $f_{n}+g_{n} \stackrel{\tau_{s}}{\longrightarrow} f+g$; et par [4] (Th. 4.2) nous avons alors $\partial\left(f_{n}+g_{n}\right) \rightarrow \partial(f+g)$.

(c) D'après la preuve de (a) et le lemme 4.3, nous avons $\cup_{\lambda \geq 0} \lambda\left(\operatorname{Dom} f_{n}-\operatorname{Dom} g_{n}\right)=X$ pour $n$ suffisamment grand. On conclut alors par [5] (Cor. 1).

Corollaire 4.1. Sous les hypothèses du théorème 4.2, nous avons $\max \left(f_{n}, g_{n}\right) \stackrel{A W}{\longrightarrow} \max (f, g)$ et ces fonctions sont convexes propres et sci à partir d'un certain rang.

Démonstration. Posons $h_{n}=\max \left(f_{n}, g_{n}\right)$ et $h=\max (f, g)$. Nous avons epi $h_{n}=$ epi $f_{n} \cap$ epi $g_{n}$ et epi $h=$ epi $f \cap$ epi $g$. Comme $f$ est quasi-continue et $\cup_{\lambda \geq 0} \lambda$ (Dom $f-\operatorname{Dom} g$ ) $=X$, il est facile de voir que epi $f$ est

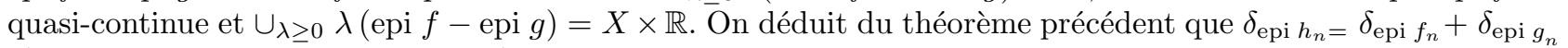
$\stackrel{A W}{\longrightarrow} \delta_{\text {epi } f}+\delta_{\text {epi } g}=\delta_{\text {epi } h}$ i.e. $h_{n} \stackrel{A W}{\longrightarrow} h$ et ces fonctions sont convexes propres et sci. 
Comme conséquence du théorème 4.2, on retrouve aussi les résultats de stabilité de Beer et Lucchetti [17] puis ceux de McLinden et Bergstrom [31] :

Corollaire 4.2 ([17], Th. 3.4). Soient $X$ un Banach et $f_{n}, f, g_{n}, g$ des fonctions convexes propres sci définies sur $X$ avec $f_{n} \stackrel{A W}{\longrightarrow} f$ et $g_{n} \stackrel{A W}{\longrightarrow} g$. Supposons que $f$ soit finie et continue en un point du domaine de $g$. Alors $f_{n}+g_{n} \stackrel{A W}{\longrightarrow} f+g$ et ces fonctions sont propres.

Démonstration. $f$ est quasi-continue car elle est continue. D'autre part, la continuité de $f$ en un point du domaine de $g$ implique que Dom $f-$ Dom $g$ est un voisinage de 0 , donc absorbant; par suite $\cup_{\lambda \geq 0} \lambda$ (Dom $f-$ Dom $g)=X$. On conclut alors avec le théorème 4.2 .

Corollaire 4.3 ([31], Th. 5). Supposons que $\operatorname{dim} X<+\infty$ et $f_{n}, f, g_{n}, g$ des fonctions convexes propres sci telles que $f_{n} \stackrel{e}{\longrightarrow} f$ et $g_{n} \stackrel{e}{\longrightarrow} g$. Si $0 \in \operatorname{int}\left(\operatorname{Dom} f-\operatorname{Dom} g\right.$ ), alors $f_{n}+g_{n} \stackrel{e}{\longrightarrow} f+g$ et ces fonctions sont propres.

Démonstration. En dimension finie, il est classique de vérifier que la quasi-continuité d'une fonction convexe est toujours satisfaite et que la condition $0 \in \operatorname{int}(\operatorname{Dom} f-\operatorname{Dom} g)$ équivaut à $\cup_{\lambda \geq 0} \lambda(\operatorname{Dom} f-\operatorname{Dom} g)=X$. Dans ce cas aussi, la convergence au sens d'Attouch-Wets cö̈ncide avec l'épi-convergence [6]. On conclut alors avec le théorème 4.2 .

Remarque 4.1. i) Une généralisation de ce dernier corollaire en dimension finie se trouve dans la référence [33]. ii) Remarquons aussi que sous les hypothèses du corollaire 4.3, et par application du corollaire 4.1, on retrouve la proposition 7.48 de [42].

Lorsqu'on se place dans les hypothèses du corollaire 4.2, il est intéréssant de donner un résultat liant la convergence d'Attouch-Wets à la convergence continue [42]. Plus précisément nous avons :

Corollaire 4.4. Soient $X$ un espace de Banach et $f_{n}, f: X \longrightarrow \overline{\mathbb{R}}$ des fonctions convexes propres sci telles que $f_{n} \stackrel{A W}{\longrightarrow} f$. Si $f$ est finie et continue en un point $x \in X$ alors $\forall x_{n} \rightarrow x, f_{n}\left(x_{n}\right) \rightarrow f(x)$.

Démonstration. Il suffit d'appliquer le corollaire 4.2 aux fonctions $f_{n}, f$ et $g_{n}=\partial_{\left\{x_{n}\right\}}, g=\partial_{\{x\}}$.

En particulier ce résultat généralise le corollaire 3B de [43] (voir aussi la Prop. 4.10 de [39]) au cas de la dimension finie. Une démonstration directe du corollaire 4.4, indépendamment des problèmes de stabilité et dans le cadre de la caractérisation de la convergence d'Attouch-Wets en termes d'approximations inf-convolutives généralisées, se trouve dans [25,34].

Remarque 4.2. La conclusion du théorème 4.2 peut tomber en défaut si on remplace la convergence au sens d'Attouch-Wets par la convergence au sens de Mosco. En effet :

Soit $X$ un espace de Hilbert de base hilbertienne $\left(x_{n}\right)_{n}$ et soit $\left(v_{n}\right)_{n}$ la suite définie par $v_{n}=\frac{1}{n}\left(x_{n}+x_{n+1}\right)$. Considérons les ensembles : $C_{n}=\left\{\alpha x_{n}+(1-\alpha) v_{n}, 0 \leq \alpha \leq 1\right\}, \quad D_{n}=\left\{x /\left\langle x, x_{n}\right\rangle=0\right\}$ et $E_{n}=$ $\operatorname{esp}\left\{x_{1}, \ldots, x_{n}\right\}$.

On vérifie aisément que $C_{n} \stackrel{M}{\longrightarrow} C=\{0\}, D_{n} \stackrel{M}{\longrightarrow} D=X$ et $E_{n} \stackrel{M}{\longrightarrow} E=X$. D'autre part, $C_{n} \cap D_{n}=$ $\emptyset \stackrel{M}{\longrightarrow} C \cap D=\{0\}$. Donc, ni la stabilité ni la propreté des $f_{n}+g_{n}$ ne sont assurées, avec $f_{n}=\delta_{C_{n}}, f=\delta_{C}$ et $g_{n}=\delta_{D_{n}}, g=0$. De même, $C_{n} \cap E_{n}=\left\{x_{n}\right\} \stackrel{M}{\longleftrightarrow} C \cap E=\{0\}$. Ici on récupère seulement la propreté des $f_{n}+h_{n}$, avec $h_{n}=\delta_{E_{n}}$ et $h=0$.

Concernant la stabilité de la somme par la convergence au sens de Mosco, et l'intérêt que présente ce problème en optimisation convexe, on peut consulter par exemple $[3,10]$.

Théorème 4.3. Soit $X$ un Banach et soient $f: X \longrightarrow \overline{\mathbb{R}}$ une fonction convexe propre sci et $C$ une partie convexe fermée non vide de $X$. Supposons que

1) $f$ ou $C$ est quasi-continue;

2) $\cup_{\lambda \geq 0} \lambda(\operatorname{Dom} f-C)=X$; 
3) $(f, C)$ est bien posé au sens de Tikhonov.

Alors, pour toute suite $\left(f_{n}\right)_{n}$ de fonctions convexes propres sci telle que $f_{n} \stackrel{A W}{\longrightarrow} f$ et pour toute suite $\left(C_{n}\right)_{n}$ de convexes fermés telle que $C_{n} \stackrel{A W}{\longrightarrow} C$, nous avons

a) $\inf \left(f_{n}, C_{n}\right) \longrightarrow \inf (f, C)$;

b) Si $x_{n} \in \varepsilon_{n}-\arg \min \left(f_{n}, C_{n}\right)$, $\varepsilon_{n} \downarrow 0$, alors $x_{n} \longrightarrow \arg \min (f, C)$.

Démonstration. Celle-ci est identique à celle du théorème 17 ([23], p. 58), sauf en remplaçant l'hypothèse de qualification : $f$ continue en un point de $C$ (ou Dom $f \cap \operatorname{int} C \neq \emptyset$ ), par les hypothèses 1 ) et 2 ) de notre théorème qui sont plus générales, et qui assurent la convergence $f_{n}+\delta_{C_{n}} \stackrel{A W}{\longrightarrow} f+\delta_{C}$.

Corollaire 4.5. Sous les même hypothèses du théorème 4.3, $(f, C)$ est bien posé au sens de Levitin-Polyak.

Démonstration. La démonstration est identique à celle du corollaire 18 ([23], p. 58), avec le même argument avancé pour justifier la preuve du théorème 4.3 .

Soient $X$ et $Y$ deux espaces de Banach, $A: X \longrightarrow Y$ un opérateur linéaire et $f: X \longrightarrow \overline{\mathbb{R}}$ et $g: Y \longrightarrow \overline{\mathbb{R}}$ deux fonctions.

On note par $A f$ et $g \circ A$, les fonctions $(A f)(y)=\inf \{f(x) / A x=y\}, y \in Y$, et $(g \circ A)(x)=g(A(x))$, $x \in X$. On désigne par $A^{*}$ l'opérateur adjoint de $A$, i.e. l'opérateur défini de $Y^{\prime}$ dans $X^{\prime}$ et vérifiant $\left(A^{*} y^{\prime}\right)(x)=$ $y^{\prime}(A x), \forall x \in X, \forall y^{\prime} \in Y^{\prime} . R(A)$ et $\operatorname{Ker} A$ désignent respectivement l'image et le noyau de $A$.

Théorème 4.4. Soient $A_{n}, A: X \longrightarrow Y$ des opérateurs linéaires continus tels que $\left\|A_{n}-A\right\| \longrightarrow 0$ et $f_{n}, f: X \longrightarrow \overline{\mathbb{R}}$ des fonctions convexes propres sci telles que $f_{n} \stackrel{A W}{\longrightarrow} f$, $f$ est fortement ou faiblement inflocalement compacte et $\operatorname{Ker} A \cap\left\{x \in X / f^{\infty}(x) \leq 0\right\}=\{0\}$. Alors

1) $A_{n} f_{n} \stackrel{A W}{\longrightarrow}$ Af et ces fonctions sont convexes ;

2) si de plus $X$ est réflexif et $f$ est faiblement inf-localement compacte, alors les fonctions $A_{n} f_{n}$, Af sont propres et sci et la borne inférieure dans ces fonctions est atteinte en tout point où elle est finie.

\section{Démonstration.}

1) Posons $B_{n}, B: X \times \mathbb{R} \longrightarrow Y \times \mathbb{R}$, les opérateurs linéaires continus définis par $B_{n}(x, \alpha)=\left(A_{n} x, \alpha\right)$ et $B(x, \alpha)=(A x, \alpha)$. On vérifie aisément que $\left\|B_{n}-B\right\|=\left\|A_{n}-A\right\| \longrightarrow 0$. D'autre part, la condition Ker $A \cap$ $\left\{x \in X / f^{\infty}(x) \leq 0\right\}=\{0\}$ est équivalente à $\operatorname{Ker} B \cap(\text { epi } f)^{\infty}=\{0\}$. Les hypothèses du théorème 3.1 sont satisfaites, donc $B_{n}$ (epi $\left.f_{n}\right) \stackrel{A W}{\longrightarrow} B($ epi $f)$. Comme $B_{n}\left(\right.$ epi $\left.f_{n}\right) \subset$ epi $\left(A_{n} f_{n}\right) \subset \overline{B_{n}\left(\text { epi } f_{n}\right)}$, alors $\overline{\text { epi }\left(A_{n} f_{n}\right)}$ $\stackrel{A W}{\longrightarrow} \overline{\text { epi }(A f)}$. Ainsi, epi $\left(\overline{A_{n} f_{n}}\right) \stackrel{A W}{\longrightarrow}$ epi $(A f)$, i.e. $\overline{A_{n} f_{n}} \stackrel{A W}{\longrightarrow} A f$, ou encore $A_{n} f_{n} \stackrel{A W}{\longrightarrow} A f$. La convexité de ces fonctions résulte d'une vérification classique.

2) Commençons d'abord par vérifier que $A f$ et $A_{n} f_{n}$ sont propres. En effet, soient $x_{0} \in$ Dom $f$ et $y_{0}=A x_{0}$. Nous avons $(A f)\left(y_{0}\right) \leq f\left(x_{0}\right)<+\infty$. Donc $A f$ n'est pas identiquement égale à $+\infty$. Supposons que $(A f)(y)=-\infty$ pour un certain $y$, et soit $\left(x_{n}\right)_{n}$ une suite telle que $f\left(x_{n}\right) \longrightarrow-\infty$ et $A x_{n}=y$. On peut supposer sans nuire à la généralité, que $(0,0) \in$ epi $f$. Soit $j$ la jauge d'un voisinage $V$ de 0 , convexe fermé équilibré tel que $V \cap$ epi $f$ soit un compact, et soit $\alpha \in \mathbb{R}$. Pour $n$ suffisamment grand, $z_{n}=\left(x_{n}, \alpha\right) \in$ epi $f$. Si $\left(j\left(z_{n}\right)\right)_{n}$ est bornée, la suite $\left(x_{n}\right)_{n}$ admet d'après le lemme 3.3 une sous-suite qui converge vers un élément $x$, et par semi-continuité inférieure $f(x)=-\infty$. Ceci contredit la propreté de $f$. Si $\left(j\left(z_{n}\right)\right)_{n}$ n'est pas bornée, on peut supposer que $j\left(z_{n}\right) \longrightarrow+\infty$. D'après le lemme 3.3, la suite $\left(\frac{z_{n}}{j\left(z_{n}\right)}\right)_{n}$ converge pour une sous-suite vers un élément non nul $(x, 0) \in(\text { epi } f)^{\infty}$. Il en résulte que $f^{\infty}(x) \leq 0$ et $A x=0$. Ainsi, $x \in \operatorname{Ker} A \cap\left\{t / f^{\infty}(t) \leq 0\right\}=\{0\}$, ce qui est absurde. Donc $A f$ est propre. Il en est de même pour $A_{n} f_{n}$ car pour $n$ suffisamment grand, nous avons $\operatorname{Ker} A_{n} \cap\left\{x / f_{n}^{\infty}(x) \leq 0\right\}=\{0\}$ et $f_{n}$ est faiblement inf-localement compacte. La semi-continuité inférieure de $A f$ et de $A_{n} f_{n}$ est une conséquence immédiate du théorème 3.1 et des égalités, epi $(A f)=B($ epi $f)$ et epi $\left(A_{n} f_{n}\right)=B_{n}$ (epi $\left.f_{n}\right)$. Le reste suit classiquement par de légères modifications au niveau de la preuve de la propreté des fonctions $A_{n} f_{n}$ et $A f$. 
Corollaire 4.6. Supposons que $\operatorname{dim} X<+\infty$, et soient $A_{n}, A: X \longrightarrow Y$ des opérateurs linéaires continus tels que $\left|A_{n}(x)-A(x)\right| \longrightarrow 0, \forall x \in X$ et $f_{n}, f: X \longrightarrow \overline{\mathbb{R}}$ des fonctions convexes propres sci telles que $f_{n} \stackrel{e}{\longrightarrow} f$ et $\operatorname{Ker} A \cap\left\{x \in X / f^{\infty}(x) \leq 0\right\}=\{0\}$. Alors $A_{n} f_{n} \stackrel{A W}{\longrightarrow}$ Af et ces fonctions sont convexes propres sci.

En particulier, on retrouve le résultat de stabilité de McLinden et Bergstrom ([31], Th. 7) lorsque $Y$ est aussi de dimension finie.

Théorème 4.5. Soient $A_{n}, A: X \longrightarrow Y$ des opérateurs linéaires continus tels que $\left\|A_{n}-A\right\| \longrightarrow 0$ et $g_{n}, g: Y \longrightarrow \overline{\mathbb{R}}$ des fonctions convexes propres sci telles que

1) $g$ est quasi-continue et $g_{n} \stackrel{A W}{\longrightarrow} g$;

2) $\cup_{\lambda \geq 0} \lambda(R(A)-\operatorname{Dom} g)=Y$.

Alors $g_{n} \circ A_{n}$ et $g \circ A$ sont convexes propres sci et $g_{n} \circ A_{n} \stackrel{A W}{\longrightarrow} g \circ A$.

Lemme 4.5. Soit $g: Y \longrightarrow \overline{\mathbb{R}}$ une fonction convexe propre sci et quasi-continue. Les propriétés suivantes sont équivalentes :

1) $\operatorname{Ker} A^{*} \cap\left\{t / g^{* \infty}(t) \leq 0\right\}=\{0\}$;

2) $R(A)^{\perp} \cap\left\{t / g^{* \infty}(t) \leq 0\right\}=\{0\}$;

3) $\cup_{\lambda \geq 0} \lambda(\overline{R(A)}-\operatorname{Dom} g)=Y$;

4) $\cup_{\lambda \geq 0} \lambda(R(A)-\operatorname{Dom} g)=Y$.

Démonstration. L'équivalence entre 1) et 2) est évidente puisque $\operatorname{Ker} A^{*}=R(A)^{\perp}$. Pour montrer que 2) est équivalente à 3$)$, il suffit de remarquer que la condition 2) est équivalente à $F=\left\{t / g^{* \infty}(t)+f^{* \infty}(-t) \leq 0\right\}=$ $\{0\}$, avec $f=\delta \overline{R(A)}$ et d'appliquer le lemme 4.3. La vérification de l'implication 4$) \Longrightarrow 2$ ) est classique. Il suffit de prendre les conjuguées des fonctions indicatrices des deux membres de 4$)$ et de se rappeler que $g^{* \infty}=\left(\delta_{\text {Dom } g}\right)^{*}$.

$2) \Longrightarrow 4)$ : soit $M=\cup_{\lambda \geq 0} \lambda(\operatorname{Dom} g-R(A))$. On a $\left(\delta_{M}\right)^{*}=\delta_{F}$ et $\delta_{\bar{M}}=\left(\delta_{M}\right)^{* *}=\left(\delta_{F}\right)^{*}=0$. Il en résulte que $\bar{M}=Y$, et par un raisonnement analogue à celui utilisé dans la preuve de l'implication 4) $\Longrightarrow 5$ ) du lemme 4.3 , on montre que $M=Y$.

Démonstration du théorème 4.5. Il est clair que les opérateurs $A_{n}^{*}$ et $A^{*}$ sont fortement et faiblement $\left(\sigma\left(Y^{\prime}, Y\right)\right.$, $\left.\sigma\left(X^{\prime}, X\right)\right)$ continus et que $\left\|A_{n}^{*}-A^{*}\right\| \longrightarrow 0$. D'autre part, nous avons $g_{n}^{*} \stackrel{A W}{\longrightarrow} g^{*}$ et $g^{*}$ est $\sigma\left(Y^{\prime}, Y\right)$ inflocalement compacte. La condition 2) est équivalente d'après le lemme 4.5, à $\operatorname{Ker} A^{*} \cap\left\{t / g^{* \infty}(t) \leq 0\right\}=\{0\}$. Par un raisonnement analogue à celui qui est utilisé dans la preuve du théorème 4.4 (moyennant le Th. 3.3), on montre que $A_{n}^{*} g_{n}^{*} \stackrel{A W}{\longrightarrow} A^{*} g^{*}$, et ces fonctions sont convexes propres $\sigma\left(X^{\prime}, X\right)$ sci. Comme $A_{n}^{*} g_{n}^{*}, A^{*} g^{*}$ ne sont pas identiquement égales à $+\infty$, alors $\left(A_{n}^{*} g_{n}^{*}\right)^{*}=g_{n} \circ A_{n}$ et $\left(A^{*} g^{*}\right)^{*}=g \circ A$. Il en résulte que ces fonctions sont convexes propres et sci (voir [24,30]). Comme $\left(g_{n} \circ A_{n}\right)^{*}=\left(A_{n}^{*} g_{n}^{*}\right)^{* *}=A_{n}^{*} g_{n}^{*} \stackrel{A W}{\longrightarrow} A^{*} g^{*}=\left(A^{*} g^{*}\right)^{* *}=(g \circ A)^{*}$, alors $g_{n} \circ A_{n} \stackrel{A W}{\longrightarrow} g \circ A$ d'après le lemme 4.4 .

Lorsque $X$ et $Y$ sont de dimension finie on retrouve en particulier le théorème 8 de la référence [31].

\section{Application des résultats de stabilité À la CONVERGence D'un schéma DE DUALITÉ EN PROGRAMMATION MATHÉMATIQUE}

Dans cette section, nous appliquons les résultats de stabilité des sections 3 et 4 à la convergence d'un schéma de dualité dans un cadre non nécessairement réflexif. Nous décrivons un modèle de problèmes primaux, duaux, dans lequel l'outil de base est la convergence au sens d'Attouch-Wets. Nous obtenons sous des hypothèses naturelles, la convergence des fonctions marginales ainsi que leurs conjuguées, ce qui conduit à la convergence de tous les objets attachés au schéma de dualité. Nous donnerons ensuite une application à la programmation mathématique en dimension finie.

Soient $X$ et $Y$ deux espaces de Banach avec $Y$ séparable et $A, A_{n}: X \longrightarrow Y$ des opérateurs linéaires continus tels que $\left\|A_{n}-A\right\| \longrightarrow 0$. Soient $f_{n}, f: X \longrightarrow \overline{\mathbb{R}}$ et $g_{n}, g: Y \longrightarrow \overline{\mathbb{R}}$ des fonctions convexes propres et sci telles 
que $f_{n} \stackrel{A W}{\longrightarrow} f$ et $g_{n} \stackrel{A W}{\longrightarrow} g$. On considère les fonctions de perturbations définies par

$$
\Phi_{n}(x, y)=f_{n}(x)+g_{n}\left(A_{n} x-y\right), \quad \Phi(x, y)=f(x)+g(A x-y) .
$$

La présentation de ces fonctions sous cette forme a un intérêt fort important dans les applications (voir $[24,30]$ ). Les fonctions marginales $h_{n}, h$ associées respectivement à $\Phi_{n}$ et $\Phi$ sont définies par $h_{n}(y)=\inf _{x} \Phi_{n}(x, y), h(y)$ $=\inf _{x} \Phi(x, y)$ On considère alors les problèmes d'optimisation dits primaux :

$$
\left(\mathcal{P}_{n}\right): \inf _{x} \Phi_{n}(x, 0), \quad(\mathcal{P}): \inf _{x} \Phi(x, 0)
$$

auxquels on associe leurs problèmes duaux définis par

$$
\begin{aligned}
& \left(\mathcal{P}_{n}^{*}\right): \quad \sup _{y^{\prime}}\left\{-h_{n}^{*}\left(y^{\prime}\right)\right\}=\sup _{y^{\prime}}\left\{-\Phi_{n}^{*}\left(0, y^{\prime}\right)\right\}=\sup _{y^{\prime}}\left\{-f_{n}^{*}\left(A_{n}^{*} y^{\prime}\right)-g_{n}^{*}\left(-y^{\prime}\right)\right\}, \\
& \left(\mathcal{P}^{*}\right): \quad \sup _{y^{\prime}}\left\{-h^{*}\left(y^{\prime}\right)\right\}=\sup _{y^{\prime}}\left\{-\Phi^{*}\left(0, y^{\prime}\right)\right\}=\sup _{y^{\prime}}\left\{-f^{*}\left(A^{*} y^{\prime}\right)-g^{*}\left(-y^{\prime}\right)\right\},
\end{aligned}
$$

où $A_{n}^{*}$ et $A^{*}$ désignent respectivement les opérateurs adjoints $\operatorname{de} A_{n}$ et $A$. Nous avons toujours $\sup \mathcal{P} \mathcal{P}^{*} \leq \inf \mathcal{P}$. Le problème $(\mathcal{P})$ est dit normal si $h(0)$ est fini et $h$ est sci en 0 , ce qui est encore équivalent à inf $\mathcal{P}=\sup \mathcal{P}^{*}$ et ce nombre est fini [24]. $(\mathcal{P})$ est dit stable si $h(0)$ est fini et $h$ est sous-différentiable en 0 , i.e. $\partial h(0) \neq \emptyset$. Ceci est encore équivalent au fait que $(\mathcal{P})$ est normal et $\left(\mathcal{P}^{*}\right)$ possède des solutions [24]. Pour plus de détails sur la théorie de la dualité, on peut consulter $[24,30]$.

Le problème que nous posons est le suivant : sous quelles conditions les problèmes $(\mathcal{P})$ et $\left(\mathcal{P}^{*}\right)$ admettent-ils des solutions que l'on peut "approcher" par des solutions des problèmes "approchés" $\left(\mathcal{P}_{n}\right)$ et $\left(\mathcal{P}_{n}^{*}\right)$ ? La réponse est donnée par :

Théorème 5.1. On suppose en plus des données et des hypothèses ci-dessus que

a) $\Phi_{n}$, $\Phi$ sont fortement ou faiblement inf-localement compactes ;

b) $g$ est quasi-continue et $h$ est finie et continue en 0 ; Alors

c) $\exists \rho_{0} \in \mathbb{R}$ tel que $\left\{x / f(x)+g(A x) \leq \rho_{0}\right\}$ soit non vide et borné.

1. $(\mathcal{P})$ est stable et admet des solutions;

2. $\left(\mathcal{P}_{n}\right)$ est stable et admet des solutions; de plus $\inf \mathcal{P}_{n} \longrightarrow \inf \mathcal{P}$;

3. si $x_{n}$ est une solution de $\mathcal{P}_{n}$, et $y_{n}^{*}$ une solution de $\mathcal{P}_{n}^{*}$, la suite $\left(x_{n}\right)_{n}$ admet une valeur d'adhérence solution de $(\mathcal{P})$ et $\left(y_{n}^{*}\right)_{n}$ admet une valeur d'adhérence solution de $\left(\mathcal{P}^{*}\right)$.

Pour la preuve, nous utiliserons les lemmes suivants :

Lemme 5.1 ( $[21])$. Soient $X$ un espace vectoriel topologique localement convexe séparé et $C$ un convexe fermé non vide localement compact tel que $C^{\infty}=\{0\}$. Alors $C$ est compact.

Lemme 5.2. Sous l'hypothèse, $g$ est quasi continue, nous avons $\Phi_{n} \stackrel{A W}{\longrightarrow} \Phi$ et ces fonctions sont convexes propres et sci.

Démonstration. Considérons les opérateurs $B_{n}:(x, y) \in X \times Y \longrightarrow A_{n} x-y, B:(x, y) \in X \times Y \longrightarrow A x-y$ et les fonctions $G_{n}=g_{n} \circ B, G=g \circ B, F_{n}(x, y)=f_{n}(x)$ et $F(x, y)=f(x)$. Montrons que :

- $G_{n} \stackrel{A W}{\longrightarrow} G$ : il suffit de vérifier que les hypothèses du théorème 4.5 sont satisfaites. En effet, $g$ est quasi-continue $\left\|B_{n}-B\right\| \longrightarrow 0$ et $R(B)=R(A)-Y=Y$. Par suite, $R(B)-$ Dom $g=Y$;

- $F_{n}+G_{n} \stackrel{A W}{\longrightarrow} F+G$ : vérifions que toutes les hypothèses du théorème 4.2 sont satisfaites. En effet, il est facile de voir que

$$
\text { aff }(\operatorname{Dom} G)=\{(x, A x-z) / x \in X \text { et } z \in \text { aff }(\operatorname{Dom} g)\}
$$


et que l'application linéaire :

$$
\overline{(x, y)} \in X \times Y / \text { aff }(\operatorname{Dom} G) \longrightarrow \overline{B(x, y)} \in Y / \text { aff }(\operatorname{Dom} g)
$$

est un isomorphisme. Donc, aff (Dom $G$ ) est de codimension finie.

D'autre part, on vérifie aisément que, si $u_{0} \in \operatorname{ir}(\operatorname{Dom} g)$, alors $z_{0}=\left(0,-u_{0}\right) \in \operatorname{ir}$ (Dom $G$ ) et que la restriction de $G$ à af $f(\operatorname{Dom} G)$ est continue en $z_{0}$. Il en résulte que la restriction de $G$ à af $f$ (Dom $G$ ) est continue en tout point de $\operatorname{ir}(\operatorname{Dom} G)$ [30]. Ainsi, $G$ est quasi-continue. La seconde condition du théorème 4.2 est aussi satisfaite car Dom $F-\operatorname{Dom} G=\operatorname{Dom} f \times Y-\{(x, A x-u) / x \in X, u \in \operatorname{Dom} g\}=X \times Y$. Enfin, la convergence $\operatorname{des} F_{n}$ vers $F$ s'obtient par une vérification classique. Ainsi, $\Phi_{n} \stackrel{A W}{\longrightarrow} \Phi$ et ces fonctions sont convexes propres et sci.

Lemme 5.3. Sous les hypothèses du théorème 5.1, on a $h_{n} \stackrel{A W}{\longrightarrow} h$ et ces fonctions sont convexes propres et sci.

Démonstration. Considérons l'opérateur $A:(x, y, \alpha) \in X \times Y \times \mathbb{R} \longrightarrow(y, \alpha) \in Y \times \mathbb{R}$, et vérifions que les hypothèses du théorème 3.1 sont satisfaites avec $A_{n}=A, C_{n}=\operatorname{epi} \Phi_{n}$ et $C=\operatorname{epi} \Phi$.

D'après le lemme $5.2, C_{n} \stackrel{A W}{\longrightarrow} C$, et $C$ est fortement ou faiblement localement compact par la proposition 4.1. D'autre part, $u=(x, y, \alpha) \in \operatorname{Ker} A \cap(\operatorname{epi} \Phi)^{\infty}$ si et seulement si $y=0, \alpha=0$ et $\Phi^{\infty}(x, 0) \leq 0$. Comme $h(0)$ est fini, il existe au moins un élément $x_{0}$ tel que $\Phi\left(x_{0}, 0\right)$ soit fini. Par suite,

$$
\Phi^{\infty}(x, 0)=\lim _{\alpha \longrightarrow+\infty} \frac{\Phi\left(x_{0}+\alpha x, 0\right)-\Phi\left(x_{0}, 0\right)}{\alpha}=(f+g o A)^{\infty}(x) .
$$

Ainsi, $x \in\left\{t /(f+g o A)^{\infty}(t) \leq 0\right\}$. Mais, d'après c) et $\left([41]\right.$, Th. 3.B) l'ensemble $\left\{t /(f+g o A)^{\infty}(t) \leq 0\right\}$ est réduit au singleton $\{0\}$. Donc $x=0$ et Ker $A \cap(\text { epi } \Phi)^{\infty}=\{0\}$. Nous avons donc par le théorème 3.1, $A\left(\right.$ epi $\left.\Phi_{n}\right) \stackrel{A W}{\longrightarrow} A($ epi $\Phi)$ et $A\left(\right.$ epi $\left.\Phi_{n}\right)$ est fermé pour $n$ suffisamment grand. Comme en plus $A\left(\right.$ epi $\left.\Phi_{n}\right) \subset$ epi $h_{n} \subset \overline{A\left(\operatorname{epi} \Phi_{n}\right)}$ et $A($ epi $\Phi) \subset$ epi $h \subset \overline{A(\text { epi } \Phi)}$, alors $h_{n}$ et $h$ sont convexes sci et $h_{n} \stackrel{A W}{\longrightarrow} h$. Il existe donc une suite $\left(y_{n}\right)_{n}$ telle que $h_{n}\left(y_{n}\right) \longrightarrow h(0)$. On en déduit que $h_{n}\left(y_{n}\right)$ est fini pour $n$ suffisamment grand. Donc $h_{n}$ est propre.

\section{Démonstration du théorème 5.1.}

1. Comme $h$ est continue en $0, \partial h(0)$ est non vide et le problème $(\mathcal{P})$ est stable [24]. Soit $\rho \in \mathbb{R}$ tel que $A_{\rho}=\{x /(f+g o A)(x) \leq \rho\}$ soit non vide. Le fermé $A_{\rho} \times\{0\}$ est contenu dans $\{(x, y) / \Phi(x, y) \leq \rho\}$ qui est localement compact. Donc $A_{\rho}$ est localement compact. Mais d'après c), ([40], p. 70) et [41] (Th. 3.B), nous avons $A_{\rho}^{\infty}=\{0\}$; donc $A_{\rho}$ est compact par le lemme 5.1. La fonction $f+g o A$ est donc inf-compacte, atteint son minimum en au moins un point de $X$.

2. Comme $h$ est finie et continue en 0 , alors $h^{*}$ est $\sigma\left(Y^{\prime}, Y\right)$ inf-compacte [30]. Donc il existe au moins un nombre $\alpha>\inf h^{*}$ tel que $\left\{y^{\prime} / h^{*}\left(y^{\prime}\right) \leq \alpha\right\}$ soit non vide et borné. D'autre part, les lemmes 5.3 et 4.4 impliquent $h_{n}^{*} \stackrel{A W}{\longrightarrow} h^{*}$. Il en résulte que $\inf h_{n}^{*} \longrightarrow \inf h^{*}[16]$, i.e. $h_{n}(0) \longrightarrow h(0)$ ou encore inf $\mathcal{P}_{n} \longrightarrow \inf \mathcal{P}$. On en déduit que $h_{n}(0)$ est fini pour $n$ assez grand. Comme en plus $h_{n}$ est sci, alors le problème $\left(\mathcal{P}_{n}\right)$ est normal. Mais pour $n$ suffisamment grand, Ker $A \cap\left(\operatorname{epi} \Phi_{n}\right)^{\infty}=\{0\}$ d'après le théorème 3.1. Donc $\left\{x /\left(f_{n}+g_{n} o A_{n}\right)^{\infty}(x) \leq 0\right\}=$ $\{0\}$. Comme $\Phi_{n}$ est inf-localement compacte, on déduit, comme en 1., que $f_{n}+g_{n} o A_{n}$ est inf-compacte, et $\left(\mathcal{P}_{n}\right)$ admet au moins une solution. Montrons maintenant que $\left(\mathcal{P}_{n}^{*}\right)$ admet au moins une solution : soit $\rho>0$ tel que $\frac{\rho}{2}>\max \left(-h(0),-\inf _{n} h_{n}(0)\right)$ et $\left\{y^{\prime} / h^{*}\left(y^{\prime}\right) \leq \rho\right\}$ soit non vide. Comme $h_{n}^{*} \stackrel{A W}{\longrightarrow} h^{*}$, alors $D_{n, \rho}=$ $\left\{y^{\prime} / h_{n}^{*}\left(y^{\prime}\right) \leq \rho\right\} \stackrel{A W}{\longrightarrow} D_{\rho}=\left\{y^{\prime} / h^{*}\left(y^{\prime}\right) \leq \rho\right\}[16]$. Mais compte tenu du fait que $D_{\rho}$ est borné, les ensembles $D_{n, \rho}$ sont donc uniformément bornés [16] i.e. il existe $r>0$ tel que $D_{n, \rho} \subset \mathcal{B}_{Y^{\prime}}(0, r)$ pour $n$ suffisamment grand. Soit $\left(z_{p}^{*}\right)_{p}$ une suite telle que $h_{n}^{*}\left(z_{p}^{*}\right) \leq \inf h_{n}^{*}+\frac{1}{p}$. Pour $p$ assez grand, $z_{p}^{*} \in \mathcal{B}_{Y^{\prime}}(0, r)$. La suite $\left(z_{p}^{*}\right)_{p}$ admet donc une sous-suite qui converge $\sigma\left(Y^{\prime}, Y\right)$ vers un élément $z_{n}^{*}$ réalisant le minimum de $h_{n}^{*}$ sur $Y^{\prime}$, i.e. $z_{n}^{*}$ est une solution de $\left(\mathcal{P}_{n}^{*}\right)$.

3. Soit $y_{n}^{*}$ une solution de $\left(\mathcal{P}_{n}^{*}\right)$. Pour $n$ suffisamment grand, $y_{n}^{*} \in D_{n, \rho}$. Il en résulte que la suite $\left(y_{n}^{*}\right)_{n}$ est bornée et $y_{n}^{*} \stackrel{\sigma\left(Y^{\prime}, Y\right)}{\longrightarrow} y^{*}$ pour une sous-suite. Comme la suite $\left(y_{n}^{*}, h_{n}^{*}\left(y_{n}^{*}\right)\right)_{n}$ est bornée et que epi $h_{n}^{*} \stackrel{A W}{\longrightarrow}$ epi $h^{*}$, 
il existe une suite $\left(t_{n}^{*}, \alpha_{n}\right)_{n}$ de epi $h^{*}$ telle que $\left|y_{n}^{*}-t_{n}^{*}\right| \longrightarrow 0$ et $\left|h_{n}^{*}\left(y_{n}^{*}\right)-\alpha_{n}\right| \longrightarrow 0$. Par suite, $t_{n}^{*} \stackrel{\sigma\left(Y^{\prime}, Y\right)}{\longrightarrow} y^{*}$ pour une sous-suite et $\alpha_{n} \longrightarrow-h(0)$. Ainsi, $h^{*}\left(y^{*}\right) \leq \liminf h^{*}\left(t_{n}^{*}\right) \leq \liminf \alpha_{n}=-h(0)=\inf h^{*}$. Donc $y^{*}$ est solution de $\left(\mathcal{P}^{*}\right)$.

Soit maintenant $\left(x_{n}\right)_{n}$ une suite telle que $\Phi_{n}\left(x_{n}, 0\right) \leq h_{n}(0)+\frac{1}{n}$, et soit $M>0$ tel que $\frac{M}{2}>\sup h_{n}(0)$. Pour $n$ assez grand, nous avons $\left(x_{n}, 0, M\right) \in$ epi $\Phi_{n}$. D'autre part, le lemme 3.5 implique l'existence de deux nombres $\alpha$ et $\beta$ strictement positifs tels que $\left|A\left(x_{n}, 0, M\right)\right| \geq \alpha j\left(x_{n}, 0, M\right)-\beta$. Par suite, $j\left(x_{n}, 0, M\right) \leq \max \left(\frac{\beta+M}{\alpha}, 2\right)=\gamma$. Mais selon un argument déjà utilisé dans la section 3 , la suite $u_{n}=\left(x_{n}, 0, M\right)$ admet au moins une soussuite convergente. Il en résulte que $x_{n} \rightarrow x$ pour une sous-suite. Comme $\Phi_{n} \stackrel{M}{\longrightarrow} \Phi$ (voir [23], p. 56), alors $\Phi(x, 0) \leq \liminf \Phi_{n}\left(x_{n}, 0\right) \leq \liminf h_{n}(0)=h(0)$, i.e. $x$ est une solution de $(\mathcal{P})$.

Dans le cas de la dimension finie, on obtient en particulier :

Corollaire 5.1. Soient $f_{n}, f, g_{n}^{i}, g^{i}, i=1, \ldots, k$ des fonctions convexes propres et sci, définies sur $\mathbb{R}^{p}$ telles que

(a) $f_{n} \stackrel{e}{\longrightarrow} f, g_{n}^{i} \stackrel{e}{\longrightarrow} g^{i}, \forall i=1, \ldots, k$;

(b) $\exists x_{0} \in \mathbb{R}^{p}$ tel que $f\left(x_{0}\right)<+\infty, g^{i}\left(x_{0}\right)<0$ et $g^{i}$ est continue en $x_{0}, \forall i=1, \ldots, k$;

(c) $\lim _{\|x\| \underset{x \in B}{\longrightarrow}+\infty} f(x)=+\infty$ où $B:=\left\{x \in \mathbb{R}^{p} / g^{i}(x) \leq 0, \forall i=1, \ldots, k\right\}$.

On considère les problèmes :

$$
\left(\mathcal{P}_{n}\right): \inf _{x} \Phi_{n}(x, 0), \quad\left(\mathcal{P}_{n}^{*}\right): \sup _{y^{\prime}}\left\{-\Phi_{n}^{*}\left(0, y^{\prime}\right)\right\}
$$

où $\Phi_{n}$ est la fonction définie par :

$\left.\left.\Phi_{n}: \mathbb{R}^{p} \times \mathbb{R}^{p} \times \mathbb{R}^{k} \rightarrow\right]-\infty,+\infty\right]$

$$
(x, y) \rightsquigarrow f_{n}(x)+g_{n}(A x-y) \text { avec } g_{n}(x, y)=\delta_{D_{n}(x,-y)}, D_{n}=\left\{(x, y) \in \mathbb{R}^{p} \times \mathbb{R}^{k} / y=\left(y_{1}, \ldots, y_{k}\right)\right.
$$

et $\left.g_{n}^{i}(x) \leq y_{i}, \forall i=1, \ldots, k\right\}$ et $A: x \in \mathbb{R}^{p} \longrightarrow(x, 0) \in \mathbb{R}^{p} \times \mathbb{R}^{k}$.

Alors, nous avons la même conclusion que celle du théorème 5.1 avec

$$
(\mathcal{P}): \inf _{x} \Phi(x, 0),\left(\mathcal{P}^{*}\right): \sup _{y^{\prime}}\left\{-\Phi^{*}\left(0, y^{\prime}\right)\right\}
$$

où $\Phi(x, y)=f(x)+g(A x-y), g(x, y)=\delta_{D}(x,-y)$ et $D=\left\{(x, y) \in \mathbb{R}^{p} \times \mathbb{R}^{k} / y=\left(y_{1}, \ldots, y_{k}\right)\right.$ et $g^{i}(x) \leq y_{i}$, $\forall i=1, \ldots, k\}$.

Démonstration. Il suffit de vérifier que toutes les hypothèses du théorème 5.1 sont satisfaites :

1. $g_{n}$ est convexe propre et sci : comme $g_{n}^{i} \stackrel{e}{\longrightarrow} g^{i}$ et $x_{0} \in \operatorname{int}\left(\operatorname{Dom} g^{i}\right)$, alors $g_{n}^{i}\left(x_{0}\right) \longrightarrow g^{i}\left(x_{0}\right)$ [43]. Donc $D_{n}$ est non vide pour $n$ suffisamment grand. De plus, cet ensemble est convexe et fermé car $g_{n}^{i}$ est convexe et sci;

2. $g_{n} \stackrel{e}{\longrightarrow} g$ : considérons les ensembles :

$$
D_{n}^{i}=\left\{(x, y) \in \mathbb{R}^{p} \times \mathbb{R}^{k} / g_{n}^{i}(x) \leq y_{i}\right\}, D^{i}=\left\{(x, y) \in \mathbb{R}^{p} \times \mathbb{R}^{k} / g^{i}(x) \leq y_{i}\right\} .
$$

On vérifie aisément que les fonctions $h_{n}^{i}(x, y)=g_{n}^{i}(x)-y_{i}$ épi-convergent vers la fonction $h^{i}(x, y)=$ $g^{i}(x)-y_{i}$. La fonction $h^{i}$ étant convexe et inf $h^{i} \leq h^{i}\left(x_{0}, 0\right)<0$, donc $\left\{(x, y) / h_{n}^{i}(x, y) \leq 0\right\} \longrightarrow\{(x, y) /$ $\left.h^{i}(x, y) \leq 0\right\}$ (voir [49]), i.e. $D_{n}^{i} \rightarrow D^{i}$. Comme $\left(x_{0}, 0\right) \in \operatorname{int} D^{i}$ pour tout $i=1, \ldots, k$, alors $\cap_{i=1}^{k} D_{n}^{i} \longrightarrow$ $\cap_{i=1}^{k} D^{i}$ d'après [31]. Ainsi, $D_{n} \rightarrow D$ et $g_{n} \stackrel{e}{\longrightarrow} g$;

3. $h(y)=\inf _{x} \Phi(x, y)$ est finie continue en $0: f$ étant propre sci et inf-compacte sur le fermé $B$, atteint son minimum en un point $t_{0}$ de $B$. Donc $h(0)=\inf _{x \in B} f(x)=f\left(t_{0}\right) \leq f\left(x_{0}\right)$. Ainsi $h(0) \in \mathbb{R}$. Comme $g^{i}$ est continue en $x_{0}$ et $g^{i}\left(x_{0}\right)<0$, il existe un réel $a<0$ et $\varepsilon_{0}>0$ tels que $g^{i}\left(x_{0}-x\right) \leq a$ dès que $\|x\| \leq \varepsilon_{0}, x \in \mathbb{R}^{p}$ et $i=1, \ldots, k$. Posons $z:=(x, y)$ avec $\|x\| \leq \varepsilon_{0}$ et $\|y\|=\max _{i}\left|y_{i}\right| \leq-a$. Alors

$$
h(z) \leq \Phi\left(x_{0}, z\right)=f\left(x_{0}\right)+g\left(A x_{0}-z\right)=f\left(x_{0}\right)+\delta_{D}\left(x_{0}-x, y\right)=f\left(x_{0}\right) .
$$


La fonction convexe $h$ est majorée sur un voisinage de 0 est donc continue en ce point.

Commentaires sur le corollaire 5.1. Remarquons qu'on peut donner une démonstration directe du corollaire 5.1, en utilisant certains résultats du chapitre 7 de la référence [42]. En effet, nous savons que $g_{n} \stackrel{e}{\longrightarrow} g$ et ces fonctions sont convexes propres sci. L'hypothèse (b) implique que $g$ est finie et continue en $A x_{0}$. Ainsi, int Dom $g \cap R(A) \neq \emptyset$ et donc $g_{n} O A \stackrel{e}{\longrightarrow} g o A$ et ces fonctions sont convexes propres sci d'après [31]. Comme de plus $f_{n} \stackrel{e}{\longrightarrow} f$ et $x_{0} \in \operatorname{Dom} f$ alors $f_{n}+g_{n} o A \stackrel{e}{\longrightarrow} f+g o A$ et ces fonctions sont convexes propres sci [31, 42]. Or l'hypothèse (c) est équivalente au fait que $f+g o A$ est inf-compacte. On déduit de 7.32.c [42] ou directement par [47] que les fonctions $f_{n}+g_{n} o A$ sont uniformément inf-compactes pour $\mathrm{n}$ suffisamment grand; et par le théorème 7.33 de [42] $\left(\mathcal{P}_{n}\right)$ et $(\mathcal{P})$ admettent des solutions et inf $\mathcal{P}_{n} \rightarrow \inf \mathcal{P}$. De plus, si $x_{n}$ est une solution de $\left(\mathcal{P}_{n}\right)$ alors la suite $\left(x_{n}\right)_{n}$ est bornée et toute valeur d'adhérence de cette suite est une soltution de $(\mathcal{P})$.

Maintenant d'après le lemme $5.3, h_{n} \stackrel{e}{\longrightarrow} h$ et donc $h_{n}^{*} \stackrel{e}{\longrightarrow} h^{*}[1,48,49]$. Comme $h$ est fini et continue en 0 , alors $h^{*}$ est inf-compacte [30] et donc les $h_{n}^{*}$ sont uniformément inf-compactes pour $n$ suffisamment grand. Et comme précédemment, on obtient la même conclusion pour $\left(\mathcal{P}_{n}^{*}\right)$ et $\left(\mathcal{P}^{*}\right)$. De plus, nous avons $\sup \mathcal{P}_{n}^{*}=$ $h_{n}^{* *}(o)=h_{n}(0)=\inf \mathcal{P}_{n}$ pour $n$ suffisamment grand; et de la même façon $\sup \mathcal{P}^{*}=\inf \mathcal{P}^{*}$.

\section{RÉFÉRENCES}

[1] H. Attouch, Variational convergence for functions and operators. Pitman, London, Appl. Math. Ser. (1984).

[2] H. Attouch et D. Aze, Regularization and approximation of sets and functions in Hilbert spaces, dans Séminaire d'Analyse Numérique, Paper XI. Université Paul Sabatier de Toulouse (1987-1988).

[3] H. Attouch, D. Aze et R.J.-B. Wets, Convergence of convex-concave saddle functions : Continuity properties of the LegendreFenchel transform and applications to convex programming. Ann. Inst. H. Poincaré Anal. Non linéaire 5 (1988) 537-572.

[4] H. Attouch et G. Beer, On the convergence of subdifferentials of convex functions. Arch. Math. 60 (1993) 389-400.

[5] H. Attouch et H. Brezis, Duality for the sum of convex functions in general Banach spaces, Publications AVAMAC. Université de Perpignan, Nos. 84-10. Av. (1984).

[6] H. Attouch et R.J.-B. Wets, Quantitative stability of variational systems : I. The epigraphical distance. Trans. Amer. Math. Soc. 328 (1991) 695-729.

[7] H. Attouch et R.J.-B. Wets, Quantitative stability of variational systems : II. A framework for nonlinear conditionning, IIASA working paper 88-9. Laxemburg, Austria (1988).

[8] H. Attouch et R.J.-B. Wets, Quantitative stability of variational systems : III. Stability of minimizers, Working paper IIASA. Laxemburg, Austria (1988).

[9] H. Attouch et R.J.-B. Wets, A quantitative approach via epigraphic distance to stability of strong local minimizers, Publications AVAMAC. Université de Perpignan (1987).

[10] D. Aze, Convergences variationnelles et dualité. Applications en calcul des variations et en programmation mathématique, Thèse de Doctorat d'État. Université de Perpignan (1986).

[11] D. Aze et J.-P. Penot, Operations on convergent families of sets and functions. Optim. 21 (1990) 521-534.

[12] B. Bank, J. Guddat, D. Klatte, B. Kummer et K. Tammer, Nonlinear parametric optimization. Akademie Verlag (1982).

[13] G. Beer, On Mosco convergence of convex sets. Bull. Austral. Math. Soc. 38 (1988) 239-253.

[14] G. Beer, Conjugate convex functions and the epi-distance topology. Proc. Amer. Math. Soc. 108 (1990) 117-126.

[15] G. Beer, The slice topology : A viable alternative to Mosco convergence in nonreflexive spaces. Nonlinear. Anal. Theo. Meth. Appl. 19 (1992) 271-290.

[16] G. Beer et R. Lucchetti, Convex optimization and the epi-distance topology. Trans. Amer. Math. Soc. 327 (1991) 795-813.

[17] G. Beer et R. Lucchetti, The epi-distance topology : Continuity and stability results with applications to convex optimization problems. Math. Oper. Res. 17 (1992) 715-726.

[18] G. Beer et M. Thera, Attouch-Wets convergence and a differential operator for convex functions. Proc. Amer. Math. Soc. 122 (1994) 851-858.

[19] N. Bourbaki, Espaces vectoriels topologiques, Chaps. 1-2. Hermann, Paris (1966).

[20] D.L. Burkholder et R.A. Wijsman, Optimum properties and admissibility of sequentiel tests. Ann. Math. Statist. 34 (1963) $1-17$.

[21] C. Castaing et M. Valadier, Convex analysis and measurable multifunctions. Springer, Lecture Notes in Math. 580 (1977).

[22] J. Dieudonne, Sur la séparation des ensembles convexes. Math. Annal. 163 (1966) 1-3.

[23] A.L. Dontchev et T. Zolezzi, Well-posed optimization problems. Springer-Verlag, Berlin, Lecture Notes in Math. 1543 (1993).

[24] I. Ekeland et R. Temam, Analyse convexe et problèmes variationnels. Dunod, Paris (1974).

[25] K. El Hajioui, Convergences variationnelles : approximations inf-convolutives généralisées, stabilité et optimisation dans les espaces non réflexifs, Thèse Nationale. Kénitra (2002).

[26] K. El Hajioui et D. Mentagui, Slice convergence : stabilité et optimisation dans les espaces non réflexifs. Preprint. 
[27] J. Garsoux, Espaces vectoriels topologiques et distributions. Dunod, Paris (1963).

[28] J.L. Joly, Une famille de topologies et de convergences sur l'ensemble des fonctionnelles convexes, Thèse d'État. Grenoble (1970).

[29] K. Kuratowski, Topology, Vol. I. Academic Press, New York (1966).

[30] P.J. Laurent, Approximation et optimisation. Hermann (1972).

[31] L. McLinden et R. Bergstrom, Preservation of convergence of convex sets and functions in finite dimensions. Trans. Amer. Math. Soc. 268 (1981) 127-142.

[32] D. Mentagui, Stability results of a class of well-posed optimization problems. Optim. 36 (1996) $119-138$.

[33] D. Mentagui, Stabilité de l'épi-convergence en dimension finie. Pub. Inst. Math. 59 (1996) 161-168.

[34] D. Mentagui et K. El Hajioui, Convergences des fonctions convexes et approximations inf-convolutives généralisées. Pub. Inst. Math. (à paraître).

[35] J.J. Moreau, Proximité et dualité dans un espace Hilbertien. Bull. Soc. Math. France 93 (1965) $273-299$.

[36] U. Mosco, Approximation of the solutions of some variational inequalities. Ann. Scuola Normale Sup. Pisa 21 (1967) 373-394.

[37] U. Mosco, Convergence of convex sets and of solutions of variational inequalities. Adv. in Math. 3 (1969) $510-585$.

[38] U. Mosco, On the continuity of the Young-Fenchel transform. J. Math. Anal. Appl. 35 (1971) 518-535.

[39] R. Robert, Convergences de fonctionnelles convexes. J. Math. Anal. Appl. 45 (1974) 533-555.

[40] R.T. Rockafellar, Convex Analysis. Princeton University Press (1970).

[41] R.T. Rockafellar, Level sets and continuity of conjugate convex functions. Trans. Amer. Math. Soc. 123 (1966) 46-63.

[42] R.T. Rockafellar et R.J.-B. Wets, Variational analysis. Springer (1998).

[43] G. Salinetti et R.J.-B. Wets, On the relations between two types of convergence for convex functions. J. Math. Anal. Appl. 60 (1977) 211-226.

[44] Y. Sonntag, Convergence au sens de Mosco : théorie et applications à l'approximation des solutions d'inéquations, Thèse d'État. Université de Provence, Marseille (1982).

[45] Y. Sonntag et C. Zalinescu, Set convergences : An attempt of classification. Trans. Amer. Math. Soc. 340 (1993) 199-226.

[46] B. Van Cutsem, Problems of convergence in stochastic linear programming, dans Techniques of optimization, édité par Balakrishnan. Academic Press, New York (1972) 445-454.

[47] R.J.-B. Wets, A formula for the level sets of epi-limits and some applications. Mathematical theories of optimization, édité par J.P. Cecconi et T. Zolezzi. Springer, Lecture Notes in Math. 983 (1983).

[48] R.A. Wijsman, Convergence of sequences of convex sets, cones and functions. Bull. Amer. Math. Soc. 70 (1964) 186-188.

[49] R.A. Wijsman, Convergence of sequences of convex sets, cones and functions II. Trans. Amer. Math. Soc. 123 (1966) 32-45. 